\title{
REVIEW ARTICLE OPEN Impact of intracellular innate immune receptors on immunometabolism
}

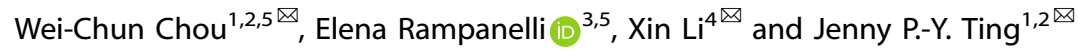

(c) The Author(s) 2021

Immunometabolism, which is the metabolic reprogramming of anaerobic glycolysis, oxidative phosphorylation, and metabolite synthesis upon immune cell activation, has gained importance as a regulator of the homeostasis, activation, proliferation, and differentiation of innate and adaptive immune cell subsets that function as key factors in immunity. Metabolic changes in epithelial and other stromal cells in response to different stimulatory signals are also crucial in infection, inflammation, cancer, autoimmune diseases, and metabolic disorders. The crosstalk between the PI3K-AKT-mTOR and LKB1-AMPK signaling pathways is critical for modulating both immune and nonimmune cell metabolism. The bidirectional interaction between immune cells and metabolism is a topic of intense study. Toll-like receptors (TLRs), cytokine receptors, and T and B cell receptors have been shown to activate multiple downstream metabolic pathways. However, how intracellular innate immune sensors/receptors intersect with metabolic pathways is less well understood. The goal of this review is to examine the link between immunometabolism and the functions of several intracellular innate immune sensors or receptors, such as nucleotide-binding and leucine-rich repeat-containing receptors (NLRs, or NOD-like receptors), absent in melanoma 2 (AIM2)-like receptors (ALRs), and the cyclic dinucleotide receptor stimulator of interferon genes (STING). We will focus on recent advances and describe the impact of these intracellular innate immune receptors on multiple metabolic pathways. Whenever appropriate, this review will provide a brief contextual connection to pathogenic infections, autoimmune diseases, cancers, metabolic disorders, and/or inflammatory bowel diseases.

Keywords: Immunometabolism; NLRs; NLRP3/AIM2 inflammasomes; innate sensors/receptors; STING; AKT-mTOR

Cellular \& Molecular Immunology (2022) 19:337-351; https://doi.org/10.1038/s41423-021-00780-y

\section{INTRODUCTION}

A growing number of studies have highlighted the intricate relationship between intracellular metabolism and inflammation. The immunometabolism field focuses on the alterations in intracellular metabolism that accompany immune cell activation and control immune cell functions. Upon activation, immune cells undergo extensive metabolic reprogramming to fulfill the drastic increase in energy demand and support immune cell functions, such as robust cytokine production, rapid proliferation, and migratory activities [1]. In particular, the increased requirement for glucose and the upregulation of the rate of aerobic glycolysis are key components of the intracellular metabolic switch induced by proinflammatory signals, which lead to a shift in glucose metabolism toward the production of lactate rather than the mitochondrial tricarboxylic acid (TCA) cycle (Fig. 1). Although extensive studies have shown the importance of metabolic reprogramming in both $T$ and $B$ cell receptor (TCR/BCR) signaling, as well as membrane-associated Toll-like receptors (TLRs), less is known about the role of intracellular innate immune receptors in immunometabolism. The purpose of this review is to highlight some key findings that reveal how innate immune receptor signaling intersects with immunometabolism.

In immunometabolism, the mitochondrial TCA cycle is coupled to oxidative phosphorylation (OXPHOS) and is a highly effective energy generation process; however, glycolysis can be more rapidly enhanced to meet the energetic demands of activated proinflammatory immune cells [2] (Fig. 1). This enhancement occurs via the upregulation of glycolytic enzymes, such as hexokinase 1 and 2 (HK1, HK2), glyceraldehyde-3-phosphate dehydrogenase (GAPDH), and pyruvate kinase isoenzyme M2 (PKM2), and via an increase in the surface expression of the glucose transporter GLUT1 [3-6]. Moreover, glycolysis fuels the pentose phosphate pathway (PPP), which amplifies the production of glycolysis-derived intermediates and is crucial in supporting proliferation and immune effector functions. In fact, the PPP generates biosynthetic precursors for nucleotides, amino acids, and fatty acid synthesis (FAS), thereby supporting anabolic growth and cytokine secretion $[1,7]$. Furthermore, the NADPH produced

\footnotetext{
'Lineberger Comprehensive Cancer Center, University of North Carolina at Chapel Hill, Chapel Hill, NC 27599, USA. ${ }^{2}$ Department of Genetics, University of North Carolina at Chapel Hill, Chapel Hill, NC 27599, USA. ${ }^{3}$ Amsterdam UMC (University Medical Center, location AMC), Department of Experimental Vascular Medicine, AGEM (Amsterdam Gastroenterology Endocrinology Metabolism) Institute, Meibergdreef 9, 1105 AZ Amsterdam, The Netherlands. ${ }^{4}$ Comparative Immunology Research Center, College of Veterinary Medicine, China Agricultural University, Beijing 100193, China. ${ }^{5}$ These authors contributed equally: Wei-Chun Chou, Elena Rampanelli. 丝mail: wcchou@email.unc.edu; xinli2021@cau.edu.cn; jenny_ting@med.unc.edu
} 

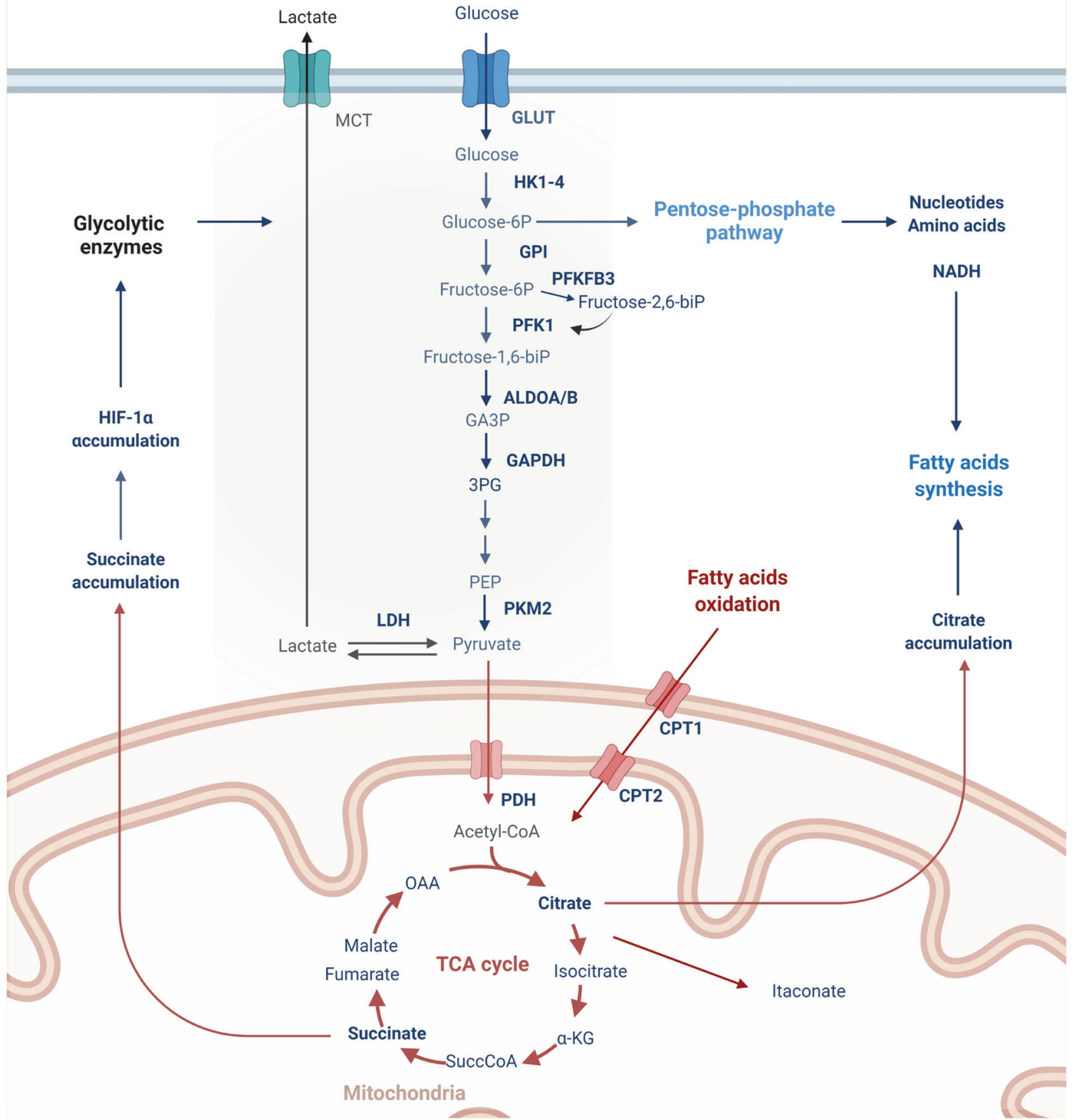

Fig. 1 Glycolysis (light green box) supports the anabolic demands of activated immune cells. Upon immune activation, glycolysis shifts pyruvate toward lactate production instead of feeding the TCA cycle. Accumulation of the TCA intermediate succinate promotes glycolysis via HIF-1 $\alpha$ stabilization. In addition, the accumulated citrate serves as a precursor for the anti-inflammatory metabolite itaconate. Glycolysis also fuels the pentose phosphate pathway (PPP) to generate nucleotides, amino acids, and NADPH, which is used with citrate for fatty acid synthesis. Oxidized fatty acids drive mitochondrial oxidative metabolism, which is more prominent in tolerogenic immune cells than in other cells

in the PPP is used for the rapid production of microbicidal reactive oxygen species (ROS) by NADPH oxidase, as well as for glutathione biosynthesis, which counteracts oxidative stress [8].

Engagement of immune receptors such as TLRs, TCR/BCR, or interleukin-2 receptor (IL-2R) dictates the metabolic switch in immune cells. Notably, these immune receptors activate the NF-KB (nuclear factor kappa-light-chain-enhancer of activated B cells) pathway and the transcription factors HIF-1a (hypoxia-inducible factor-1a) and c-Myc, thereby inducing transcriptional reprogramming toward glycolytic gene expression [3, 9-11]. Additionally, receptor-mediated activation of kinase signalings, such as the Akt (also known as protein kinase B) and mTOR (mechanistic target of rapamycin) pathways, largely mediates the high demand for cellular nutrients by sustaining glucose uptake and glycolysis, inducing de novo synthesis of fatty acids, lipids, and cholesterol, and promoting glutamine anaplerosis and protein translation [12]. 
mTOR is a master regulator of intracellular metabolism and immune cell activation and constitutes the catalytic subunit of the distinct multiprotein complexes mTORC1 and mTORC2 (mTOR complexes 1 and 2, respectively). mTORC1 is activated by the phosphatidylinositol 3-kinase (PI3K)/Akt pathway, whereas mTORC2 controls Akt activation by inducing Akt phosphorylation. Both complexes contribute to metabolic reprogramming: mTORC1 facilitates glycolysis, glutaminolysis, and protein and lipid synthesis and inhibits autophagy, while mTORC2 activates Akt, which promotes GLUT1 surface expression and aerobic glycolysis. By modulating downstream effectors, such as S6K1, 4EBP1, and Akt, these complexes regulate cell growth, proliferation, and aerobic glycolysis in response to growth factors, nutrients, and receptor signaling [12-14].

The marked dependence on glycolysis has been largely studied in classically activated M1 macrophages, monocytes, and effector T-helper 1 and 17 (Th1/17) cells [3, 6, 9, 11, 15], but glycolysis is a hallmark of all activated immune cells, including activated dendritic cells (DCs), neutrophils, B cells, and natural killer cells [9, 16-19]. Conversely, energy production via OXPHOS is more prominently associated with the longevity of quiescent homeostatic immune cells, such as naïve and memory B/T cells [20-23], and cells with immunotolerogenic properties, such as alternatively activated M2 macrophages and regulatory (Treg) or exhausted T cells [24-27]. Mitochondrial oxidative metabolism is fueled by aerobic glycolysis through the conversion of the glycolytic endproduct pyruvate into acetyl-CoA and by mitochondrial fatty acid oxidation (FAO), which generates large amounts of acetyl-CoA, $\mathrm{NADH}$, and $\mathrm{FADH}_{2}$ [1]. Acetyl-CoA is used in the TCA cycle; NADH and $\mathrm{FADH}_{2}$ are used in the electron transport chain (ETC) to produce ATP [1]. Mitochondria-dependent catabolic metabolism is largely regulated by AMPK (AMP-activated protein kinase), which is a central regulator of energy balance that is activated by energy stress (such as low AMP/ADP-to-ATP ratio, glucose/glutamine deprivation) and by the kinases LKB1 (liver kinase B1) and CaMKK2 (calcium calmodulin kinase 2). AMPK favors cellular catabolism by promoting mitochondrial fitness and suppressing anabolic processes [28]. Moreover, AMPK drives mitochondrial biogenesis and fission, as well as the clearance of damaged mitochondria via mitophagy/autophagy. Conversely, AMPK inhibits the enzymes acetyl-CoA carboxylase and HMG-CoA reductase, which are crucial in lipid and cholesterol synthesis, respectively, and inhibits mTORC1 activity $[28,29]$. Hence, by suppressing anabolism and mTOR, AMPK inhibits metabolic rewiring and limits immune cell activation.

Adding to the complexity of this intricate immunometabolic balance, the choice of energy source serves specialized immune phenotypes and specific immune functions. For example, Tregs are less reliant on glycolysis than Th1/2/17 cells, although they use glycolysis to meet the energetic demands required for migration, and glycolysis complements oxidative metabolism during expansion $[30,31]$. In addition, glycolysis was shown to enhance Foxp3 (a master regulator of Treg differentiation and immunotolerogenic functions) splicing variants containing exon 2 through the glycolytic enzyme enolase-1 [32]. Similarly, while tolerogenic M2 macrophages and Tregs are associated with increased oxidative metabolism and FAO rates, recent studies using cell-specific genetic depletion of the Cpt1a gene, which encodes the mitochondrial long-chain FA transporter carnitine palmitoyl transferase-1, have revealed that FAO is dispensable for M2 macrophages or Treg differentiation, disputing previous results obtained with the use of the CPT1 inhibitor etomoxir [6, 33-36].

During proinflammatory immune activation, the rapid increase in glycolytic flux at the expense of OXPHOS not only supports the generation of energy and biomass but also results in the accumulation of metabolic intermediates, such as succinate, itaconate, and fumarate, which may function as immunoregulatory signaling molecules (immunometabolites) [37-40]. Moreover, this metabolic repurposing has been shown to notably reshape the functional status of immune cells, particularly innate cells, through epigenetic reprogramming. The latter is tightly influenced by changes in the network of intracellular metabolic pathways and, thus, the bioavailability of metabolic intermediates, such as acetyl-coenzyme $\mathrm{A}, \mathrm{NAD}^{+}$, and a-ketoglutarate, which act as enzymatic cofactors of histone acetyltransferases, deacetylases, and histone-/DNA-demethylases, respectively [40].

Overall, a better understanding of the kinetics of immunemetabolic circuits mediated by innate immune receptor engagement can provide novel therapeutic opportunities to either boost immune defenses against pathogen infections or harness excessive pathologic inflammation in chronic inflammatory metabolic diseases. Next, we will examine the impact of several intracellular innate immune receptors/sensors (NLRs, AIM2, and STING) and immunometabolism.

\section{NLRS AND IMMUNOMETABOLISM}

The NLR (nucleotide binding domain leucine-rich repeat or nucleotide-oligomerization domain (NOD)-like receptors) family is a large group of evolutionarily conserved sensors or receptors that recognize pathogens and homeostatic alterations [41]. All members have a nucleotide-binding domain and a leucine-rich repeat (LRR) domain. Members have divergent functions including the transcriptional activation of major histocompatibility genes, inflammasome activation, regulation of cell death and modification of cell signals such as NFKB and MAPK. NLR family members are important immune defense mechanisms that sense pathogenassociated molecular patterns and danger-associated molecular patterns. Accumulating evidence has shown the pivotal roles of these intracellular immune receptors/sensors, particularly the NLRP3 inflammasome, in sensing systemic metabolic perturbations. Other NLRs have recently been shown to modulate intracellular metabolic pathways that shape the phenotype of immune cells. Although the experimental evidence is far from complete, we will summarize and discuss the recent findings on the roles of NLRP3, NLRX1, and NLRC3 in metabolic reprogramming.

\section{NLRP3 inflammasome}

As a critical component of innate immunity, the inflammasome is a large multimeric protein complex consisting of the cytosolic sensor NLR, the adapter ASC (apoptosis-associated speck-like protein containing a C-terminal caspase recruitment domain) and the effector procaspase-1 [42]. In addition, NEK7, a member of the NIMA-related kinase family, was recently identified as an NLRP3binding component that was essential for NLRP3 inflammasome oligomerization and activation [43-45]. The NLRP3 inflammasome is the most prominent inflammasome and promotes innate and adaptive immune responses. The inflammasome is generally activated in "wo steps". The first signal (priming) triggers the NFKB-mediated upregulation of inactive pro-IL-1 $\beta$ and NLRP3 (or, in some cases, nontranscriptional priming) $[42,46]$, as well as posttranslational modifications, such as ubiquitylation, phosphorylation, sumoylation, neddylation, acetylation, ADP-ribosylation and nitrosylation $[42,47-54]$. The second signal consists of the engagement of an NLRP3 activator and drives inflammasome assembly, proximity-induced caspase- 1 autoproteolysis, and the cleavage of IL-1 $\beta, I L-18$, and gasdermin $D$, culminating in the secretion of mature active cytokines and the induction of pyroptosis, which is a form of inflammatory programmed cell death [42, 55-60]. NLRP3 acts as a guardian of intracellular homeostasis and are activated by a variety of endogenous signals $[42,61]$, including the efflux of potassium or chloride ions, intracellular calcium mobilization, ROS, cytosolic mitochondrial DNA (mtDNA) and cardiolipin, mitochondrial damage, lysosomal disruption, endoplasmic reticulum (ER) stress, trans-Golgi 


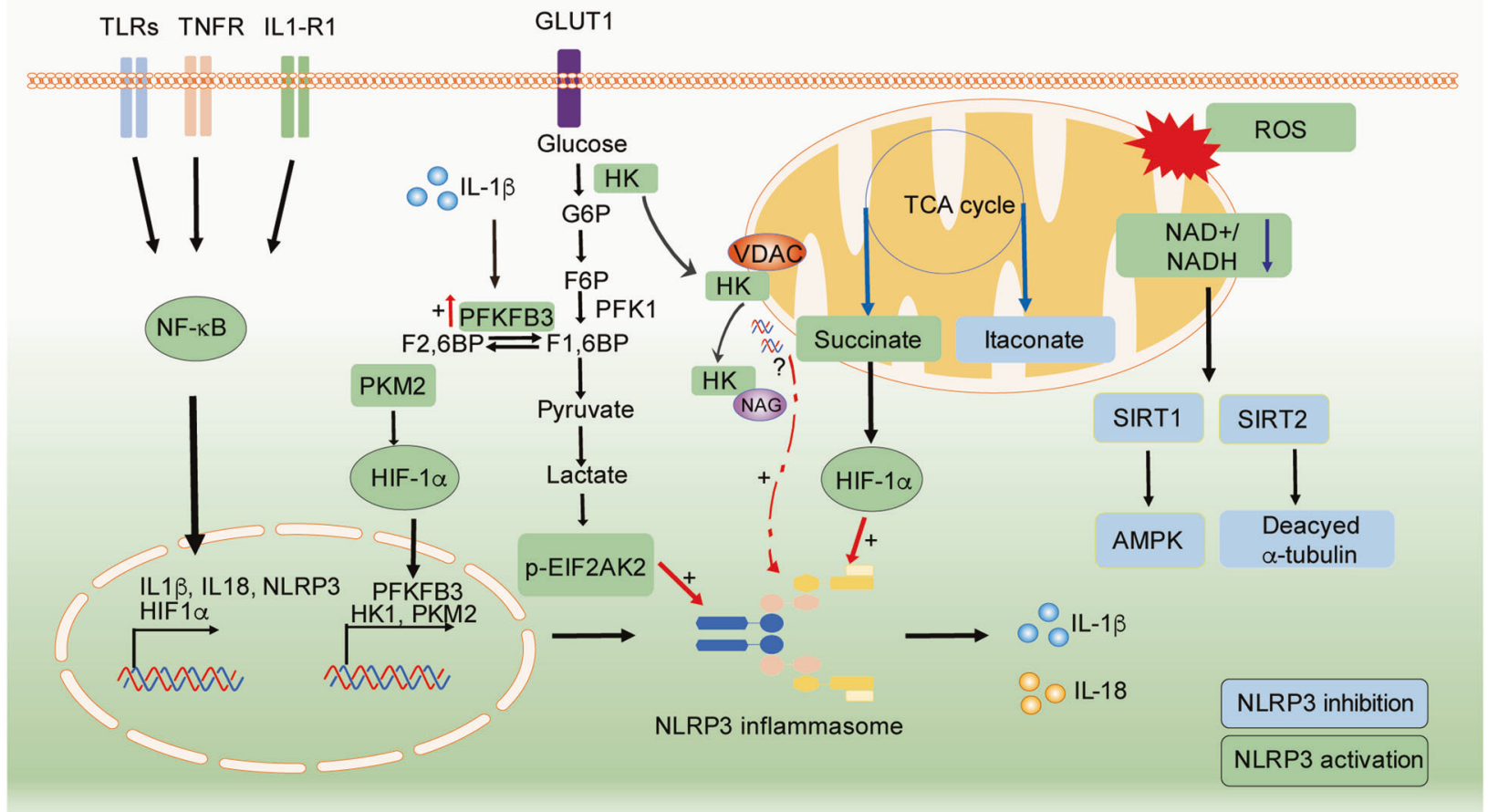

Fig. 2 Immunometabolic regulation of NLRP3 inflammasome activity. The glycolytic cascade downstream of active PKM2 drives the production of lactate, which mediates EIF2AK2 phosphorylation and enhances IL- $\beta$-mediated PFKFB3 expression to activate NLRP3. Dimeric PKM2 interacts with HIF- $1 \alpha$, thus promoting IL- $\beta$ expression and glycolysis. Bacterial NAG binds to and inhibits HK activity, which causes the dissociation of HK from VDACs and in turn releases unknown factors from mitochondria to trigger NLRP3 inflammasome activation. Aberrant mitochondrial homeostasis results in the accumulation of the TCA derivatives succinate (in green) and itaconate (in blue), which activate and suppress NLRP3, respectively. Reduced NAD ${ }^{+}$availability inactivates SIRT1/2, resulting in two activation signals for NLRP3: (i) AMPK inhibition and (ii) the deposition of acetylated $\alpha$-tubulin. Green: inducers of NLRP3 activity. Blue: inhibitors of NLRP3

disassembly, intracellular kinase signaling, and lipid uptake and accumulation [42, 46, 61]. The NLRP3 inflammasome participates in the progression of metabolic diseases, is activated by systemic metabolic disturbances (e.g., dyslipidemia and hyperglycemia) [62-71], and is a modifiable target in therapeutic approaches [72-77].

Accumulating evidence has suggested that NLRP3 activation is tightly regulated by changes in intracellular metabolic pathways, especially within immune myeloid cells (Fig. 2). Moon et al. showed that in macrophages stimulated with LPS and ATP, HK1dependent glycolysis was a critical step in NLRP3 activation. Glucose deprivation, glycolysis inhibition (by 2-DG treatment) or silencing $H k 1$ suppressed caspase- 1 activation and IL-1 $\beta$ secretion. Furthermore, activation of the Raptor/mTORC1 complex promoted the HK1-dependent increase in glycolytic flux to cause an inflammasome response in ATP-stimulated LPS-primed macrophages [4]. Consistently, Xie et al. [78] used pharmacologic inhibition and silencing of PKM2 and showed that PKM2dependent glycolysis was required for the activation of NLRP3 inflammasomes after LPS-primed macrophages were treated with ATP. Mechanistically, inflammasome activation is associated with glycolysis-driven autophagy inhibition and mitochondrial ROS (mtROS) production. In addition, PKM2-driven glycolysis results in the phosphorylation of eukaryotic translation initiation factor 2 alpha kinase 2 (EIF2AK2), which functions in the antiviral response, inflammation, and immune regulation [79] and can physically interact with NLRP3, NLRP1, NLRC4, or AIM2 [80] and promote NLRP3 activation [78, 80]. Pharmacologic or genetic inhibition of PKM2 or EIF2AK2 hampers NLRP3 inflammasome activation and protects mice from lethal endotoxemia and polymicrobial sepsis [78]. Similarly, monosodium urate and calcium pyrophosphate crystals, which are linked to gout and pseudogout flares, promote glucose uptake in macrophages and synovial fluid neutrophils, triggering glycolysis-dependent NLRP3 activation [81]. A recent study showed that IL-1 $\beta$ drives PFKFB3 expression and glycolysis in macrophages stimulated with LPS and amyloid- $\beta$, whereas inhibiting NLRP3 or PFKFB3 activity with MCC950 [82] or 3PO, respectively, inhibits glycolytic induction in stimulated macrophages [83]. Accordingly, channeling the glycolytic end-product pyruvate toward lactic acid fermentation rather than mitochondrial pyruvate oxidation is important for optimal NLRP3 activation in response to various NLRP3 agonists in macrophages. This effect appears to depend on the lactate-mediated phosphorylation of EIF2AK2, which regulates inflammasome responses [80, 84]. These findings indicate that NLRP3 may create a metabolic loop in which glycolysis is induced upstream and downstream of NLRP3 inflammasome activation.

In contrast to these findings that support the roles of glycolytic enzymes in NLRP3 activation, other studies have reported that the disruption of glycolytic flux serves as an activating signal for NLRP3. For example, Wolf et al. showed that in LPS-primed macrophages, peptidoglycan (PGN) activates NLRP3 through the release of $\mathrm{N}$-acetylglucosamine (NAG), a sugar subunit of the backbone of PGN that is produced during the lysosomal degradation of PGN. Mechanistically, both PGN and NAG inhibit the glycolytic enzyme hexokinase, thereby inducing hexokinase dissociation from the mitochondrial membrane into the cytosol. However, HK dissociation but not inhibition trigger NLRP3 inflammasome activation [85]. Moreover, although NAG treatment did not affect mitochondrial membrane integrity, it induced the cytosolic translocation of mtDNA [85], which is a known NLRP3 activator [86-88]. Accordingly, in bone marrow-derived macrophages (BMDMs), glycolytic disruption caused by the small molecule GB111- $\mathrm{NH}_{2}$ or infection with Salmonella typhimurium induced NLRP3 inflammasome formation, IL-1 $\beta$ secretion, and pyroptotic cell death via a mechanism involving mtROS production and impaired NADH formation mediated by glycolysis [89]. 
However, facilitating the TCA cycle through supplementation with the glycolytic end-product pyruvate abrogated inflammasome activation [89], indicating that the NLRP3 activating signal was downstream of glycolytic interruption. Overall, these studies indicate that while glycolysis is important for the induction of NLRP3 expression and inflammasome assembly, perturbations in the downstream TCA cycle and OXPHOS also provide the mitochondrial-derived danger signals necessary for NLRP3 activation. In addition, in contrast to inflammasome dependence on PKM2-driven glycolysis [78], the activation of PKM2 by the small molecules DASA-58 and TEPP-46 was shown to hamper the LPSinduced proinflammatory response in $M 1$ macrophages while stimulating the secretion of the anti-inflammatory M2 IL-10 cytokine in vitro [3]. Likewise, in vivo TEPP-46 administration inhibited the secretion of IL-1 $\beta$ but increased IL-10 production in LPS-induced sepsis and in a S. typhimurium model of infection [3]. This incongruence can be explained by the fact that PKM2 exists as dimers and tetramers that have opposite effects on glycolysis. PKM2 dimers can interact with HIF-1a and promote HIF-1a transcriptional activity, leading to the expression of glycolytic enzymes, as well as $\| 1 b$, whereas tetramerization of PKM2, such as in response to DASA-58 and TEPP-46 [90], impairs PKM2 nuclear translocation and association with HIF-1a [3, 91, 92]. In fact, DASA58 and TEPP-46 treatment ablate LPS-induced HIF-1a expression/ stabilization and upregulation of glycolytic rates [3].

As described above, acute inflammatory signaling favors aerobic glycolysis at the expense of mitochondrial OXPHOS. This shift results in the accumulation of TCA cycle intermediates, such as succinate. Succinate is produced in mitochondria by the succinyl-CoA enzyme and is further oxidized into fumarate by the enzyme succinate dehydrogenase (ETC complex II), thereby contributing to the pool of $m$ mROS $[2,93]$. Alternatively, succinate can be exported from the mitochondria and function as an immunometabolite in the cytosol or extracellular space [94-96]. Once in the cytosol, succinate inhibits prolyl hydroxylases and thus promotes HIF-1a stabilization and activation [94, 97], resulting in the upregulation of glycolytic enzymes [3, 11, 38, 98]. In a hallmark study, O' Neill's group identified succinate as an intracellular innate signal that accumulates in LPS-stimulated macrophages and induces HIF-1a-driven IL-1 $\beta$ upregulation. These effects were strictly dependent on the metabolic rewiring triggered by TLR4 signaling upon engagement by LPS [38]. More recently, the same group revealed that another TCA-derived immune metabolite, itaconate [39], regulated IL-1 $\beta$ by specifically blocking NLRP3 activation. Using macrophages that lacked the enzyme IRG1, which catalyzes itaconate production, and tandem mass spectrometry, the authors showed the specificity of itaconate and its cell-permeable derivative 4-octyl-itaconate in inhibiting NLRP3 activity and the occurrence of the itaconation of cysteine C548 on the NLRP3 protein [76]. The latter process is likely responsible for impairing protein-protein interactions in the inflammasome complex. The anti-inflammatory effects of 4-octylitaconate have also been reported in PBMCs isolated from CAPS (cryopyrin-associated periodic syndrome) patients and in an in vivo model of peritonitis induced by monosodium urate crystals as an NLRP3 activating signal [76]. Moreover, endogenous itaconate was recently shown to accumulate in macrophages, particularly in the context of prolonged LPS stimulation, and posttranslationally modify gasdermin $D$, thereby impairing caspase-1 processing and late NLRP3 inflammasome activation [99]. Further reports suggest an inhibitory effect of itaconate on IL-1 $1 \beta$ secretion in activated macrophages by regulating the enzymatic activity of mitochondrial SDH and glycolytic GAPDH and fructose-bisphosphate aldolase A [37, 100-102]. Consistent with the evidence that intracellular metabolites can regulate NLRP3 activation, other derivatives of TCA cycle metabolites, such as ethyl pyruvate (EP) and dimethyl fumarate (DMF), have been shown to hamper NLRP3 activation [103-105]. This suppressive effect depends on the ability of EP to attenuate mitochondrial damage and inhibit mtDNA release into the cytoplasm and by the DMF-mediated induction of the antioxidant NF-E2-related factor 2 (Nrf2), ultimately reducing $\mathrm{mtROS}$ production and cytoplasmic translocation of mtDNA [103, 105].

Mitochondria are central in NLRP3 inflammasome activation: NLRP3 is recruited to mitochondria for optimal inflammasome activity [106], and inflammasome responses are largely triggered by aberrant mitochondrial homeostasis [107]. Dysfunctional mitochondria and disturbances in OXPHOS are associated not only with mtROS generation but also the decline in $\mathrm{NAD}^{+}$levels, resulting in the inhibition of $\mathrm{NAD}^{+}$-dependent enzymes, such as sirtuins [108]. Sirtuins (SIRT1-7) are a family of evolutionarily conserved $\mathrm{NAD}^{+}$-dependent protein deacetylases that serve as key metabolic sensors and govern metabolic homeostasis, stress responses, and longevity [108]. Notably, decreased NAD ${ }^{+}$levels have been reported in LPS-primed BMDMs [89, 109, 110], suggesting the impairment of SIRT activity in primed macrophages. Specifically, SIRT1 and NLRP3 seem to mutually regulate each other: inflammasome-activated caspase-1 can cleave SIRT1 and inhibit its activity $[69,111]$, whereas SIRT1 can restrain NLRP3 activation by activating the LKB1 (liver kinase B1)/AMPK pathway, thereby promoting mitochondrial biogenesis, OXPHOS and autophagy and indirectly limiting dysfunctional mitochondria $[28,61,88]$. In addition, the activity of $\mathrm{NAD}^{+}$-dependent SIRT2 has also been shown to modulate NLRP3 activation. Misawa et al. found that inducers of NLRP3 disrupted mitochondrial homeostasis and diminished intracellular $\mathrm{NAD}^{+}$, which consequently inhibited the SIRT2-mediated deacetylation of a-tubulin. As a result, the accumulation of acetylated a-tubulin mediated mitochondrial transport to the ER, resulting in the accumulation of NLRP3 on the ER with mitochondrial ASC [109]. Moreover, He et al. demonstrated that SIRT2 prevented inflammaging by deacetylating NLRP3 and inactivating the NLRP3 inflammasome. Instead, the acetylation of NLRP3 facilitates inflammasome assembly and enhances its activity in macrophages, underscoring the link between TCA metabolite availability and regulatory posttranslational modifications of NLRP3 [53].

NLRP3 activity can be triggered by systemic metabolic perturbations, such as increased dietary lipids, which fuel oxidative catabolic metabolism [112]. For example, Wen et al. reported that the saturated fatty acid palmitate, which is highly abundant in Western diets and in the circulation of obese diabetic individuals, can activate the NLRP3 inflammasome by suppressing AMPK activity, consequently inhibiting autophagy while increasing ROS production [62]. In addition, AMPK restrains inflammation by negatively regulating mTOR complex l, which is accompanied by the suppression of protein and lipid synthesis required for mounting a proper inflammatory response [12].

While the intracellular accumulation of cholesterol and saturated fatty acids is regarded as a trigger of inflammasome assembly $[62,64,65]$, polyunsaturated FAs, such as the $\omega-3$ FA docosahexaenoic acid, attenuate NLRP3-driven inflammation $[113,114]$. In contrast to FAS, which accompanies proinflammatory functions, FAO is typical of type 2 immunity and antiinflammatory functions [2] and produces $\mathrm{NADH}, \mathrm{FADH}_{2}$, and acetyl coenzyme $A$, which are further used to generate ATP or ketone bodies under starvation conditions [1]. Interestingly, two studies showed that the main ketone metabolite betahydroxybutyrate is a potent inhibitor of NLRP3 priming and assembly in macrophages and neutrophils [77, 115]. However, another study by Moon et al. indicated that FAO could promote NLRP3 assembly, while pharmacological inhibition or genetic ablation of the enzyme NADPH oxidase 4 (NOX4) reduced FAO rates and NLRP3 activation [116]. In contrast, in a murine stroke model, administration of the NOX inhibitor apocynin resulted in neuroprotective and anti-inflammatory effects, and there was a reduction in the expression of NLRP3, ASC, caspase-1, IL-18, and $\mathrm{IL}-1 \beta$ [117]. These findings suggest that specific enzymes and metabolic pathways differentially govern the assembly and 
activation of the NLRP3 inflammasome depending on the location and underlying pathology.

Overall, these studies highlight the intricate relationship between the NLRP3 inflammasome and metabolism. Subtle alterations in one metabolic pathway can perturb associated metabolic circuits, leading to the accumulation of specific endogenous metabolites or dangerous molecules that in turn activate NLRP3 and induce inflammation (Fig. 2).

\section{NLRX1}

NLRX1 (nucleotide-binding domain and LRR-containing protein X, also known as CLR11.3, NOD5, or NOD9) was first discovered as a mitochondria-associated NLR that possesses a mitochondrial targeting sequence (MTS) encoded in the first 39 amino acids of the protein. NLRX1 consists of 975 amino acids and is ubiquitously expressed in mammalian tissues. It contains two of the three typical NLR domains (a central NACHT domain and a carboxyterminus LRR domain) and an unconventional $\mathrm{N}$-terminal domain that gives rise to the ' $X$ ' in the nomenclature [118-120]. NLRX1 was first shown to negatively regulate mitochondrial antiviral signaling protein (MAVS)-mediated type I IFN and NF-KB signaling by interacting with MAVS through its LRR domain at the mitochondrial outer membrane [119]. Furthermore, NLRX1 has also been shown to directly bind to RNA [121-123]. NLRX1 is also present in the inner mitochondrial membrane and within the matrix $[118,120]$. Internalized NLRX1 interacts with the protein UQCRC2 in the ETC and regulates the production of ROS in mitochondria [118]. Hence, it is possible that the distribution of NLRX1 in distinct cellular compartments allows it to interact with multiple molecules of cellular pathways to exert its diverse effects and modulate immune responses. NLRX1 has been linked to the regulation of the host innate immune response in the context of pathogen sensing, inflammation, ROS production, ER stress, and autophagy [121, 124-134]. One consistency is that many studies have shown that NLRX1 attenuates diseases, including chronic obstructive pulmonary disease (COPD) [135], autoimmune diseases [136-139], and cancer [140-144]. In cancer, NLRX1 has been shown to downregulate key cytokines that are important in cancer progression, such as tumor necrosis factor (TNF) and interleukin (IL)-6. Accumulating evidence indicates that NLRX1 has multiple functions $[145,146]$. In this review, we will focus on its role in regulating metabolic reprogramming.

In the immunometabolism field, NLRX1 is a bridge between inflammation and metabolism. In cancer cells, the removal of extracellular glucose or the addition of the glycolysis inhibitor 2-deoxyglucose (2-DG) significantly reduced NLRX1 expression in both primary- and SV40-transformed MEFs. This result indicates that the expression of NLRX1 is glucose-regulated [147]. In addition, NLRX1 was also shown to regulate glycolysis. In one study, knockdown of NLRX1 in HeLa cells reversed the acidification of the culture medium, suggesting that NLRX1 may contribute to the metabolic switch to glycolysis in tumor cells [144]. NLRX1 also regulates the activities of mitochondrial complex I and III to maintain ATP levels in the presence of TNF- $a$ and promote the metabolic switch toward aerobic glycolysis. Specifically, NLRX1 depletion in MDA-MB-231 cells (a triple-negative breast cancer cell line) decreased the organization and activity of OXPHOS complexes, affecting OXPHOS-dependent cell proliferation and the migration of triple-negative breast cancer cells. The loss of NLRX1 in MDA-MB-231 cells further impaired lysosomal function and mitophagy-mediated turnover of damaged mitochondria in the presence of TNF-a [148]. These studies suggest that NLRX1 functions as a critical mitochondrial protein to maintain mitochondrial metabolism for energy homeostasis and lysosomal function to regulate the invasion and metastasis capabilities of metastatic breast cancer [144, 148].

Other studies have linked NLRX1 to fatty acids and amino acid metabolites. For example, compound libraries were screened, and different lipids, such as coenzyme A-containing fatty acids and sterols, were identified that could bind to the LRR domain of NLRX1. Moreover, punicic acid (PUA), a polyunsaturated fatty acid, or docosahexaenoic acid (DHA), exerted anti-inflammatory effects and suppressed NF-KB activity in an NLRX1-dependent manner in LPS-activated BMDMs. In contrast, PUA treatment did not ameliorate experimental colitis in $N / r \times 1^{-/-}$mice compared to wild-type mice. The study further indicated that the interaction of NLRX1 and PUA was necessary for modulating mucosal immune responses and relieving inflammation in the gut [149]. Another study showed that NLRX1 protected against mitochondrial damage and oxidative stress in kidney epithelial cells and that the loss of NLRX1 promoted oxygen consumption and oxidative stress, disturbed mitochondrial morphology, and subsequently increased ROS production and apoptosis in tubular epithelial cells during renal ischemia-reperfusion injury. The NLRX1-mediated loss of ROS in tubular cells contrasts with the findings of an earlier study suggesting that NLRX1 induced ROS; hence, the effect of NLRX1 on ROS may be dependent on the experimental conditions and cell types. Of note, this study also found that the polyunsaturated fatty acid DHA, but not the saturated fatty acid palmitate, prevents apoptosis during reoxygenation in an NLRX1-dependent fashion, confirming the finding shown by Lu et al. that polyunsaturated fatty acids can modulate NLRX1 function [150].

Leber et al. showed that NIrx $1^{-1-}$ intestinal epithelial cells (IECs) showed increased expression of genes associated with amino acid metabolic pathways, specifically glutamine metabolism (Glud1, Got1, and Gpt), based on RNA-sequencing data. Moreover, NIrx $1^{-/-}$ intestinal organoids have significantly increased activity of glutamate dehydrogenase (GDH), which catalyzes the reversible conversion of the oxidative deamination of glutamate to aketoglutarate and ammonia while turning $\mathrm{NAD}(\mathrm{P})^{+}$to $\mathrm{NAD}(\mathrm{P}) \mathrm{H}$ [151] and decreases $\mathrm{NAD}^{+}$levels without changing NADH levels, indicating altered cycling or consumption of $\mathrm{NAD}^{+}$in these cells. Interestingly, oral glutamine supplementation altered the microbiome composition and alleviated the severity of inflammatory bowel disease in N/rx $1^{-/-}$mice [152]. This finding provides a link between NLRX 1 and glutamine metabolism and suggests that the administration of specific amino acids, such as glutamine, can ameliorate intestinal inflammation by modulating the microbiome in an NLRX1-dependent manner.

Interestingly, NLRX1 is also expressed by $\mathrm{CD}^{+}{ }^{+} \mathrm{T}$ cells, and the loss of NLRX1 results in increased disease severity and increased numbers of Th1 and Th17 cells producing inflammatory cytokines (IFN- $\gamma$, TNF, and IL-17) in dextran sodium sulfate-induced (DSS) colitis. Nlrx ${ }^{-1-}$ CD4+ T cells show altered metabolic behavior with increased expression of the genes Cpt1a, Fabp4, and Glut1, which are responsible for the uptake and utilization of glucose and fatty acids. Likewise, the loss of NLRX1 in $\mathrm{CD}^{+}{ }^{+} \mathrm{T}$ cells increases the rate of incomplete FAO and enhances glycolysis in Th17 cells [153]. To evaluate NLRX1 as an immunometabolic regulator of inflammation, the small molecule NX-13 (1,3,5-tris(6methylpyridin-2-yloxy)benzene was shown to bind to NLRX1. The drug was delivered orally, exhibited increased local concentrations throughout the gastrointestinal tract, including the distal parts of the colon, and selectively targeted and activated NLRX1 in CD4 ${ }^{+}$ $T$ cells in IBD. NX-13 treatment in vitro decreased the differentiation of $\mathrm{CD}^{+}{ }^{+} \mathrm{T}$ cells into Th1 and Th17 subsets increased OXPHOS and decreased NF-KB activation and ROS. NX-13 treatment in vivo reduced Th1 and Th17 subsets, enhanced Treg cells, and reduced neutrophils in high-dose DSS-induced colitis. In addition, NX-13 decreased inflammatory cytokines such as IL-8, MCP1, and IL-6, as well as NF-KB and ROS production in PBMCs from moderate-tosevere ulcerative colitis patients. These studies suggest that NX-13 is a promising NLRX1 agonist and a translational medicine candidate for treating a number of NLRX1-attenuated inflammatory conditions $[154,155]$. 

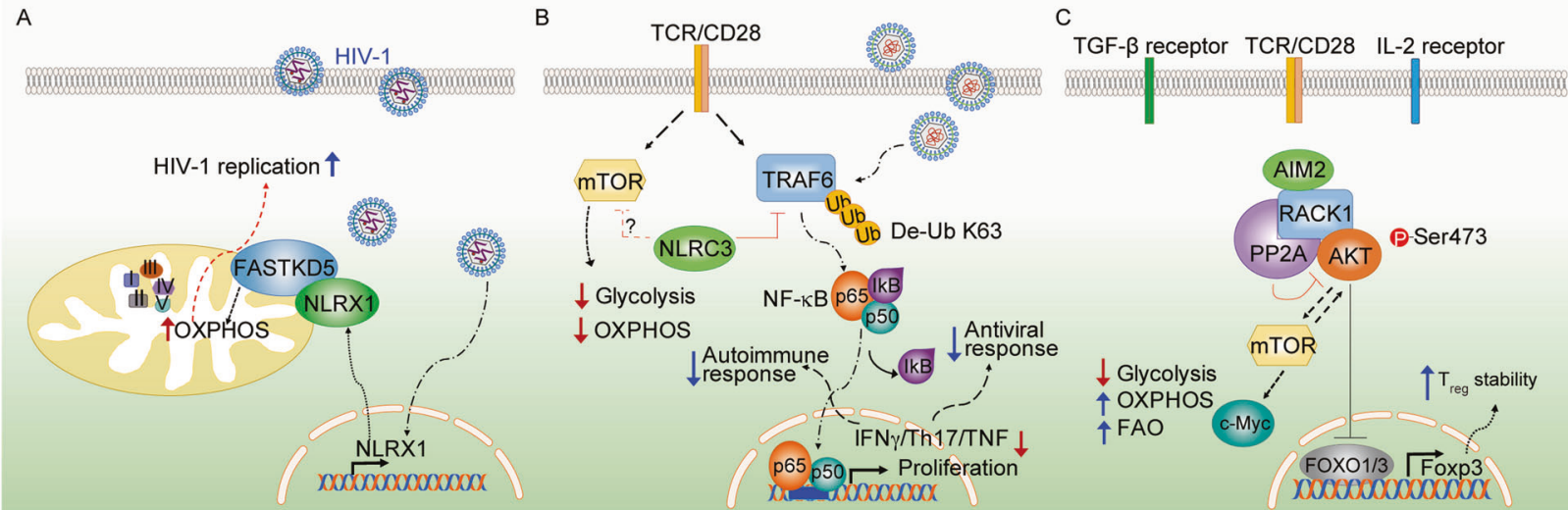

Fig. 3 Overview of intracellular innate immune receptors in T cell metabolic reprogramming. A HIV-1 infection increases the expression of NLRX1, which directly associates with FASTKD5 to promote OXPHOS and viral replication. B NLRC3 in T cells acts as an intrinsic attenuator that regulates $\mathrm{T}$ cell signaling pathways and metabolic progress to restrict autoimmune and virus-specific CD4+ T cell responses. C AIM2 facilitates the interaction between RACK1 and PP2A phosphatase, causing the dephosphorylation of AKT to restrain the activity of the mTOR pathway, thereby promoting Foxp 3 expression and $\mathrm{T}_{\text {reg }}$ cell stability

In addition to glucose metabolism, NLRX1 also regulates fatty acid metabolism. NIrx $1^{-1-}$ mice are protected against high-fat diet-induced metabolic syndrome, kidney dysfunction and the progression of nonalcoholic fatty liver disease (NAFLD). The loss of NLRX1 in hepatocytes also leads to increased FAO and decreased steatosis. Hence, NLRX1 controls nonimmune parenchymal hepatocyte energy metabolism by restricting mitochondrial fatty acid-dependent OXPHOS and enhancing glycolysis [156]. Likewise, $N / r \times 1^{-1-}$ mice are protected against high-fat diet-induced pancreatic lipid accumulation and hyperglycemia [157].

Singh et al. showed that NLRX1 is associated with Fas-activated serine-threonine kinase family protein-5 (FASTKD5) through its LRR domain and colocalized with mitochondrial RNA granules in the mitochondrial matrix. FASTKD5 negatively regulates the processing of mitochondrial transcripts to modulate the activity of complex-I and complex-IV and respiratory supercomplex formation [158]. An earlier report also identified FASTKD5 as a putative NLRX1 interaction partner using overexpressed NLRX1 as the bait for immunoprecipitation followed by mass spectrometry [159]. The interaction of NLRX1 and FASTKD5 has been further confirmed in mitochondria and has been shown to negatively regulate RNA processing, OXPHOS activity, and mitochondrial ribosome biogenesis and translation [160]. The connection of NLRX1 with FASTKD5 is also relevant to T cells, and the association of NLRX1 and FASTKD5 has been shown to enhance the expression of mitochondrial respiratory complex components in human $\mathrm{CD}^{+}{ }^{+} \mathrm{T}$ cells and promote OXPHOS and glycolysis [161]. The study showed an essential role of OXPHOS in promoting HIV-1 replication in human $\mathrm{CD}^{+}{ }^{+}$T cells, which is achieved in an NLRX1and FASTKD5-dependent manner. The study underscores the importance of NLRX1-mediated regulation of immunometabolism, which is relevant for controlling HIV-1 in CD4 ${ }^{+}$T cells [161] (Fig. $3 \mathrm{~A}$ ) and provides a rationale for the therapeutic targeting of NLRX1 or OXPHOS for HIV treatment.

In addition, NLRX1 has been shown to protect experimental autoimmune encephalomyelitis (EAE), a commonly used experimental animal model of human multiple sclerosis (MS), by attenuating microglial activation and reducing encephalitogenic T cells $[138,139,162]$. NIrx1 mRNA expression is significantly increased in $\mathrm{CD} 14^{+}$PBMCs in relapsing-remitting MS patients, and the identification of rare NLRX1 mutations in MS patients, such as p.Lys172Asn, p.Glu192Ter, and p.Arg860Trp links NLRX1 genetic variants to the incidence of MS in humans. These studies indicate that NLRX1 may be a therapeutic target for MS. A recombinant protein (dNP2-LRR), which is consistent with the blood-brain barrier permeable peptide dNP2 [163] and the LRR domain of NLRX1, alleviates EAE [164]. In summary, these studies provide a perspective on targeting NLRX1 in not only pathogen infection but also autoimmune diseases. Going forward, it will be intriguing to examine whether metabolic modulators that have shown efficacy in modulating NLRX1-dependent functions, such as NX-1 or rapamycin, can be used to mitigate diseases such as MS or COPD.

\section{NLRC3}

NLRC3 (NLR family CARD-containing 3) is also named CLR16.2 or NOD3 and consists of N-terminal caspase activation and recruitment domain, a central nucleotide-binding domain, and a C-terminal LRR domain [165]. NLRC3 was first identified in human Jurkat $\mathrm{T}$ lymphocytes, and its expression is downregulated rapidly upon stimulation with anti-CD3/anti-CD28 antibodies or treatment with PMA/ionomycin. This finding suggests that NLRC3 functions as a suppressor of T cell activation [166]. NLRC3 is predominantly expressed in both human and murine immune tissues and cells [166]. In addition, as an innate immune receptor, NLRC3 negatively regulates LPS-induced NF-KB activation downstream of TLRs by interacting with TNF receptor-associated factor 6 (TRAF6) and further altering the ubiquitination state of TRAF6 in macrophages [167]. The loss of NLRC3 was found to enhance the innate immune response to cytosolic DNA, DNA viruses, and cyclic di-GMP [168]. NLRC3 interacts with both stimulators of interferon genes (STING), which is a DNA sensor, and the protein kinase TBK1 and impairs the STING-TBK1 interaction, resulting in the subsequent reduction in downstream type I interferon production and NF-KB activation [168]. Furthermore, NLRC3 binds HSV-60 double-stranded DNA (dsDNA) mainly through its LRR domain. Viral DNA binding to NLRC3 increases its ATPase activity, which is needed to facilitate the release of STING and TBK1 and then mediate the downstream IFN-I response [123]. Tocker et al. [169] identified a scaffolding protein, IQGAP1, that specifically associates with NLRC3 and disrupts the NLRC3-STING interaction to regulate the type I IFN pathway in the cytosol of human monocytic and epithelial cells.

In addition to pathogen sensing, the expression of NLRC3 is reduced in the colorectal tumor tissues of patients compared to healthy controls [170]. Karki et al. [170] further showed that $\mathrm{NIrC3}^{-/-}$ mice are more susceptible to colitis and colorectal tumorigenesis than wild-type mice and that the oncogenic inhibitory effect of NLRC3 is more dominant in IECs. At the immunometabolism level, NIrC3 ${ }^{-1-}$ mice show increased phosphorylation of S6 kinase, 4EBP1, and AKT at Ser473, all of which are downstream targets of mTOR. 
344

Mechanistically, NLRC3 associates with the p85 subunit of PI3K, disrupting the interaction between the PI3K p85 and p110a subunits and reducing the activity of PI3K p85 itself. Additionally, deletion of the CARD, NACHT, or LRR domains of NLRC3 impairs the ability of NLRC3 to interact with either the p85 or p110a subunit of PI3K. These studies demonstrated that NLRC3 restricts the PI3K-mTOR axis during colon tumorigenesis $[170,171]$. The PI3K-mTOR axis has been shown to be a central integrator of immune cell metabolism and regulates glucose and fatty acid metabolism during homeostasis and diseases [172-175]. In CD4 ${ }^{+} \mathrm{T}$ cells, NLRC3 attenuates interferon- $\gamma$ and TNF expression by CD4 $+\mathrm{T}$ cells and reduces $\mathrm{T}$ helper 1 (Th1) and Th17 cell proliferation. Moreover, the loss of NLRC3 in T cells results in improved protection against lymphocytic choriomeningitis virus infection but worsens EAE and exacerbates Th1 and Th17 responses during $M$. tuberculosis infection [176, 177]. Interestingly, NIrc3 ${ }^{-/-} \mathrm{CD} 4+\mathrm{T}$ cells showed increased phosphorylation of 4E-BP1, which lies downstream of mTOR, and increased glycolysis and OXPHOS occurred upon TCR stimulation, enhancing the proliferation and cytokine production in $\mathrm{NIrC3}^{-1-} \mathrm{CD} 4+\mathrm{T}$ cells. Mechanistically, NLRC3 in T cells interacts with TRAF6 and negatively regulates its K63-linked ubiquitination to reduce downstream NF-KB signaling and diminishes metabolic pathways to attenuate CD4+ T cell activation and proliferation [177] (Fig. 3B). In addition, a recent study described the effect of NLRC3 on platelet-derived growth factor (PDGF)-induced proliferation in pulmonary artery smooth muscle cells (PASMCs) by inhibiting the PI3K-mTOR pathway [178]. Specifically, PDGF induced abnormal PASMC proliferation, and this effect was inhibited by both a PI3K inhibitor (Ly294002) and an mTOR inhibitor (rapamycin). Overexpression of NLRC3 suppressed the expression of PI3K and mTOR in PDGF-stimulated PASMCs. This study suggests that the NLRC3/PI3K/mTOR pathway plays a critical role in PASMC proliferation. In summary, these studies revealed the impact of NLRC3 on metabolism in different cell types to control cell proliferation and activation by modulating PI3K-mTOR signaling and NFKB-mediated pathways.

\section{ABSENT IN MELANOMA 2}

AIM2 (absent in melanoma 2) is a non-NLR molecule belonging to the AIM2-like receptor (ALR) family and is a DNA receptor that triggers inflammasome activation [179-182]. AIM2 consists of C-terminal HIN-200 and N-terminal pyrin (PYD) domains and is a cytosolic DNA receptor that can directly bind to dsDNA via the HIN-200 domain. Its PYD domain interacts with the PYD domain of the inflammasome adapter protein ASC (ASC-like protein containing a carboxy-terminal (ARD). The caspase activation and recruitment domain (CARD) of ASC bind to the CARD of procaspase-1 to form the AIM2 inflammasome, which further induces caspase- 1 activation, leading to downstream IL-1 $\beta$ and IL18 maturation and gasdermin D cleavage [183]. AIM2 recognizes dsDNA in a sequence- and structure-independent but the lengthdependent manner, which requires a minimum of 80 base pairs for optimal inflammasome activation [184]. Under steady-state conditions, AIM2 is in an autoinhibitory confirmation due to the interaction of the PYD and HIN domains, and this state is altered by dsDNA binding to the HIN domain, which further recruits ASC to interact with the free PYD domain and initiates oligomerization and inflammasome activation [184, 185]. The importance of the inflammasome-dependent role of AIM2 is observed in the sensing of microbial DNA during infectious diseases and in tumorigenesis and several inflammatory and autoimmune diseases, such as atherosclerosis, neuroinflammation, psoriasis, dermatitis, arthritis, SLE, and colitis [186-188]. Next, we will highlight the evidence linking AIM2 to immunometabolism.

Sepsis is an overreactive host immune response to microbial infection that results in dysregulated systemic inflammation accompanied by the secretion of multiple proinflammatory mediators, such as TNF, IL-1 $\beta$, and high mobility group Box 1
(HMGB1) [189]; sepsis has also been linked to immunometabolism in macrophages. Previous reports have shown that the $M 2$ isoform of pyruvate kinase muscle 2 (PKM2) regulates glycolysis to promote IL-1 $\beta$ and HMGB1 release in LPS-stimulated macrophages during sepsis pathogenesis [3, 190]. As mentioned in the NLRP3 section, Xie et al. showed that PKM2-mediated metabolic programming (glycolysis) promotes not only NLRP3 but also AIM2 inflammasome activation by producing lactose to modulate EIF2AK2 phosphorylation in macrophages during sepsis. Pharmacologic inhibition of the PKM2-EIF2AK2 pathway can reduce inflammasome activation and protect mice from endotoxemia and sepsis. In addition, the loss of PKM2 suppresses inflammasome activation, and the specific deletion of PKM2 in myeloid cells protects septic mice against death in response to the AIM2 inflammasome [78]. Thus, this study provides a rationale for the therapeutic targeting of the PKM2-EIF2AK2 axis to control inflammasome by modulating immunometabolism. In addition to PKM2-mediated glycolysis in macrophages, which promotes both NLRP3 and AIM2 inflammasome activation [78], Cho et al. recently demonstrated a novel mechanism by which activation of the AIM2 inflammasome links glucose transporter 1 (GLUT1)mediated glycolysis to regulate the acute exacerbation of lung fibrogenesis during bacterial infection [191]. Myeloid cell-specific Glut1 knockout (LysM-Cre-Glut $1^{f / f l}$ ) results in reduced morbidity and collagen levels in bleomycin-induced lung fibrosis upon Streptococcus pneumoniae infection. It also results in reduced activation of the AIM2 inflammasome by poly(dA:dT) in Glut1deficient cells in vitro [191].

In addition to innate immunity and inflammation, recent studies have revealed that AIM2 plays an essential role in cancers or autoimmune diseases in an inflammasome-independent manner by altering PI3K-AKT-mTOR signaling and immunometabolism. AIM2 was originally discovered to be a tumor suppressor gene in human melanomas [192], and reduced AIM2 expression was associated with poor prognosis in patients and AIM2 mutations in human colorectal tumors [193-195]. In colorectal cancer (CRC), Wilson et al. and Man et al. demonstrated that AIM2 suppressed azoxymethane (AOM)/DSS-induced colitis-associated cancer, as well as spontaneous CRC, in a mouse model $[196,197]$. These two independent studies showed increased tumor burdens and shortened colon lengths in Aim2 $2^{-1-}$ mice compared to wild-type mice, while the inflammasome-dependent cytokines IL-1 $\beta$ and IL-18 were intact in the Aim2 $2^{-1-}$ colon, indicating that AIM2 plays an inflammasome-independent role in the development of colon cancer. Mechanistically, AIM2 restricts proliferation but promotes cell death in colon progenitors by inhibiting AKT activation, which is the central molecule in the PI3K-mTOR pathway [196, 197]. Furthermore, AIM2 associates with and restricts the activity of DNA-dependent protein kinase (DNA-PK), a PI3K-related family member, to suppress AKT activation [196]. Similar to the finding in CRC, AIM2 expression in hepatocellular carcinoma (HCC) patient tissues was negatively correlated with HCC progression [198, 199]. In HCC cells, AIM2 overexpression inhibits HCC cell proliferation and invasion by suppressing the mTOR-S6K1 axis in an inflammasomedependent manner [198]. The PI3K-AKT-mTOR signaling pathway plays a critical role in providing nutrients for the growth of normal immune cells and tumor cells by promoting anabolic processes (glycolysis) and regulating other metabolic processes, such as protein and lipid synthesis, OXPHOS, and autophagy, which is a conserved catabolic process $[175,200]$. Hence, these studies link AIM2 with the PI3K-AKT-mTOR signaling pathway in cancer cells.

Importantly, two independent groups recently reported that AIM2 regulates AKT activation in immune cells during EAE. Ma et al. found that AIM2 negatively regulated EAE progression in an inflammasome-independent manner in microglia in both the $A$ and $B$ subtypes of EAE [201]. MOG-induced EAE can be separated into the $A$ and $B$ subtypes based on the use of low or high concentrations of complete Freund's adjuvant [202]. Similar to 


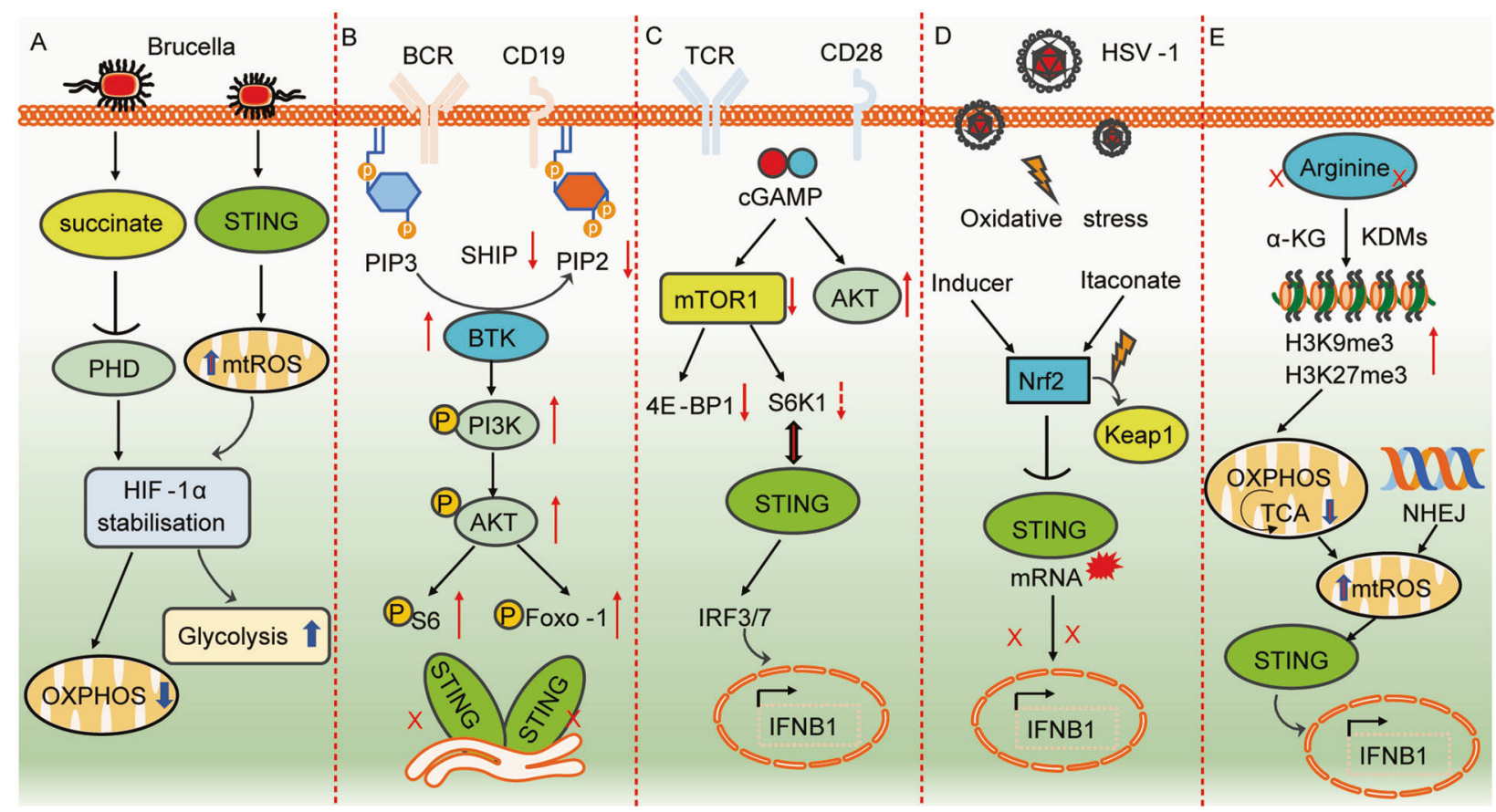

Fig. 4 STING-mediated PI3K-AKT-mTOR signaling and activation during metabolic reprogramming. A STING regulates metabolic reprogramming in macrophages via HIF-1 $\alpha$ during $B$. abortus infection. B STING interferes with BCR activation and negatively regulates CD19- and Btk-mediated PI3K signaling. C Reciprocal regulation of STING and TCR stimulation by mTORC1 leads to IFN-I production. D Nrf2 negatively regulates STING-mediated metabolic reprogramming in response to HSV-1 viral infection. E Arginine starvation induces STING activation through nuclear DNA damage via epigenetic silencing of metabolic and DNA repair genes

that in tumor cells, AIM2 limits antiviral pathway-related inflammation by interacting with DNA-PK to dissociate the DNAPK-AKT3-IRF3 complex [201]. In addition to being an innate inflammasome sensor that regulates the behavior of innate immune cells or epithelial cells during infection or inflammation, the vital role of AIM2 in controlling the function of regulatory $T$ (Treg) cells in a T cell-intrinsic but inflammasome-independent manner has been reported in EAE and T cell-mediated colitis [203]. In contrast to previous reports showing that NLRP3, ASC, and caspase-1 exacerbate EAE [204-207], AIM2 mitigates EAE without affecting IL-1 $\beta$ or IL-18 but increases the number of IL-17Aproducing $\mathrm{CD}^{+}{ }^{+} \mathrm{T}$ cells and decreases Foxp3 ${ }^{+}$Treg cells in the spinal cord. Interestingly, AIM2 is highly expressed by both human and murine Treg cells induced by TGF $\beta$, and its promoter is occupied by RUNX1, ETS1, BCL11B, and CREB, which are transcription factors that are associated with Treg cells. Both the adaptive transfer of $\mathrm{Aim}^{-1-}$ Treg cells in T cell-mediated colitis and the specific deletion of Aim2 in Treg cells show that AIM2 is required for Treg cell stability and control of autoimmune diseases [203]. Additionally, Aim2 $2^{-/}$Treg cells have increased glycolysis but reduced lipid OXPHOS and enhanced AKT-mTOR signaling, which indicates that AIM2 is essential in regulating Treg cell metabolism. Mechanistically, AIM2 associates with RACK1, which is a scaffold protein that forms a complex with PP2A and AKT, to dephosphorylate AKT and reduce AKT-mTOR signaling [203] (Fig. 3C). This study uncovered the functions of AIM2 in regulating immunometabolism in adaptive immune cells and suggests a unifying role of AIM2 in interacting with the AKT-mTOR metabolic pathway in many cell types. Thus, these studies show the unexpected role of intracellular innate immune sensors/receptors in the regulation of $\mathrm{T}$ cell immunometabolism (Fig. 3).

\section{STIMULATOR OF INTERFERON GENES}

STING (also known as TMEM173, MITA, MPYS, and ERIS) [208-211] is a universal receptor for cyclic dinucleotides, including the bacterial second messengers cyclic di-AMP, cyclic di-GMP and 3', 3'-cGAMP. In metazoans, cyclic GMP-AMP synthase (cGAS) lies upstream of STING and can bind dsDNA, resulting in $2^{\prime}, 3^{\prime}$-cGAMP synthesis. These cyclic dinucleotides bind to STING at the ER and promote STING trafficking from the ER to perinuclear puncta. During this process, STING recruits TANK binding kinase 1 (TBK1), which phosphorylates the transcription factor interferon regulatory factor 3 (IRF3), resulting in the production of type I interferons (IFNs). Recently, several studies have linked immunometabolic pathways and STING activation, which is the focus of the following discussion.

In myeloid cells, STING activation repolarizes M2 macrophages to M1-like macrophages, which are inflammatory and undergo metabolic reprogramming [212-214]. This repolarization of M2 macrophages to M1 macrophages occurs during Brucella abortus infection, and an impaired TCA cycle in M1-like macrophages drives succinate accumulation. The accumulation of succinate suppresses prolyl hydroxylase (PHD) activity, leading to HIF-1a stabilization. STING activation in M1 cells also causes increased mtROS, which similarly stabilize HIF-1a. HIF-1a then alters immunometabolism by reducing OXPHOS and increasing glycolysis (Fig. 4A). During $M$. tuberculosis infection, STING and downstream type I IFN cause metabolic reprogramming characterized by reduced glycolytic activity and OXPHOS and increased mitochondrial damage characterized by mtROS [215]. In addition to these links between STING and metabolic effectors, the mTOR downstream signal S6 kinase 1 (S6P1) forms a complex with STING and TBK1 in adenovirus-infected dendritic cells to activate IRF3 [216]. Viruses use these pathways for immune evasion. For example, the Poxviral F17 protein sequesters Raptor and Rictor in the Golgi to block CGAS-STING signaling [217].

STING also affects immunometabolism in adaptive immune cells. B cells from systemic lupus erythematosus (SLE) patients have highly activated BCRs, and STING is thought to attenuate the pathogenesis of this disease because STING expression is low in SLE patients compared to controls. In support of this conclusion, 
Sting-KO mice have increased numbers of splenic marginal zone (MZ) and germinal center (GC) B cells [218] and exhibit greater pathogenesis than WT mice. Btk-PI3K signaling plays an important role in $\mathrm{MZ}$ B cell homeostasis, and Sting-KO mice show enhanced BTK but reduced inhibitory phosphatase SHP-1 levels. Furthermore, activated pPI3K, pAkt pFoxo-1/Foxo-1, and pS6/S6 were all increased in Sting-KO B cells. However, in one study, STING did not alter PI3K recruitment or activity but directly affected the activity of $\mathrm{PI} 3 \mathrm{~K}$ by reducing $\mathrm{PI}(3,4) \mathrm{P} 2$ levels. Furthermore, $\mathrm{PI} 3 \mathrm{~K}$ inhibitors significantly decreased the colocalization of the BCR and STING. These studies suggest the bidirectional regulation of PI3K- and STING-mediated BCR signaling (Fig. 4B).

STING is expressed by T cells, resulting in the induction of type I IFN, reduced cell proliferation and increased cell death, although the latter is not observed in vivo [219]. cGAMP inhibits CD4 ${ }^{+}$T cell proliferation, resulting in a sustained G0-G1 phase of the cell cycle, which is accompanied by reduced activation of mTOR1, S6K1, and 4E-BP1 [220]. RNA profiling and pathway analysis indicated that CGAMP reduces lipid synthesis-related genes that are regulated by $\mathrm{mTORC} 1$. Using gene deletion strains, it was found that the STING-activated IRF3/7 pathway suppressed the reduction in $\mathrm{mTORC} 1$ in T cells and T cell proliferation. Conversely, mTORC1 activation is needed for STING-induced type I interferon production [220]. Blocking mTOR1 with rapamycin or deleting Raptor abolished cGAMP-mediated IFN-I production in activated $\mathrm{CD}^{+}{ }^{+} \mathrm{T}$ cells. Collectively, these findings indicate a regulatory circuit in which STING activation by cGAMP attenuates mTOR signaling, leading to reduced T cell proliferation, while mTORC1 activation via S6K1 is required for STING-mediated IFN-I production (Fig. 4C)

In other studies, glutathione peroxidase 4 (GPX4), an enzyme that balances oxidation and reduction reactions, was shown to mediate STING activation during viral infection [221]. GPX4 inhibitors can suppress IFN- $\beta$ mRNA and protein expression during HSV-1 infection in murine peritoneal macrophages and human THP1 cells [221]. Gpx4 conditional deletion results in an attenuated antiviral innate immune response due to enhanced cellular lipid peroxidation, which inhibits the cGAS-STING pathway. Increased lipid peroxidation causes the carbonylation of STING, which reduces its trafficking to the ER and blocks STING activation because STING localization to the ER is an essential step for its activation.

Others have found a link between the TCA cycle-derived metabolite itaconate (4-octyl-itaconate, 4-OI) and STING expression. Itaconate can activate the nuclear transcription factor Nrf2 (nuclear factor erythroid-derived 2-like 2) and reduce STING mRNA levels by inducing mRNA instability [222]. In addition to its in vitro relevance, itaconate can suppress STING expression and type I IFN in cells from patients with the interferonopathy and STINGassociated autoimmune disease SAVI (STING-associated vasculopathy with onset in infancy) (Fig. 4D). Interestingly, another group showed that nitro-fatty acids formed by the addition of nitrogen dioxide to unsaturated fatty acids inhibit STING palmitoylation and mitigate type I interferon production in SAVI-derived fibroblasts [223].

Finally, a recent report showed a link between arginine metabolism and STING [224]. Arginine is one of three amino acids that can activate mTOR, and some cancer cells are deficient in arginine synthesis. Arginine starvation causes the depletion of a-ketoglutarate and the inactivation of histone demethylases, resulting in the silencing of genes involved in OXPHOS and DNA repair. As a result of increased DNA damage and an increase in cytosolic DNA due to arginine starvation, cGAS-STING is activated, followed by the induction of type I IFN (Fig. 4E).

Aside from metabolic reprogramming at the cellular level, STING plays an important role in several metabolic diseases, as shown in these reviews $[225,226]$. STING exacerbates NAFLD and nonalcoholic steatohepatitis (NASH) disease models, and STING expression is higher in parenchymal liver cells from patients with NAFLD than in those from controls [227]. In mouse models of NASH and NAFLD, STING deficiency results in attenuated hepatic fibrosis, steatosis, and inflammation [228]. Mechanistically, NAFLD and obesity can result in increased release of oxidative mtDNA into the cytosol, which causes STING activation. Others observed obesity-induced cytosolic mtDNA release, which triggers the cGAS-STING pathway [229, 230]. The mitochondrial protein disulfide-bond A oxidoreductase-like protein (DsbA-L) blocks cGAS-STING by preventing mtDNA leakage and increases phosphodiesterase PDE3B/PDE4, CAMP, and PKA signaling to promote thermogenesis in adipocytes. These findings suggest that blocking the CGAS-STING pathway represents a new therapeutic approach for multiple metabolic diseases.

\section{CONCLUDING REMARKS AND FUTURE PROSPECTIVE}

Our understanding of the mechanisms governing immunometabolism has been greatly expanded in the last few years, and the studies described in this review emphasize the emerging recognition of crosstalk between intracellular innate immune receptors/sensors and metabolic pathways in shaping inflammatory responses and impacting the course of inflammatory diseases. A deeper knowledge of this intricate crosstalk can lead to novel therapeutic strategies targeting immunometabolic circuits for the treatment of metabolic and inflammatory diseases. Examples of currently approved anti-inflammatory therapies that target metabolism include metformin (targeting mTOR, used for the treatment of type 2 diabetes), rapamycin (targeting AMPK and ETC complex I, used as an immunosuppressive drug), and DMF (antioxidant and enhancer of mitochondrial respiration) [231]. However, to advance our knowledge and future therapies, it is crucial to understand the spatiotemporal immunometabolic adaptations during the course of diseases and to resolve the heterogeneity of the immunometabolic networks at the single-cell level. Great progress in single-cell technologies, such as single-cell RNA sequencing, mass cytometry, Met-Flow (flow cytometry to detect metabolic enzymes), digital spatial transcriptomic, and Cellular Indexing of Transcriptomes and Epitopes by Sequencing (CITE sequencing), can provide the required platforms for addressing these important questions [232, 233]. Using these new approaches to obtain a deeper understanding of immunometabolism mediated by innate immune receptors will greatly advance our appreciation of this still-nascent field.

\section{REFERENCES}

1. O'Neill LA, Kishton RJ, Rathmell J. A guide to immunometabolism for immunologists. Nat Rev Immunol. 2016;16:553-65

2. Loftus RM, Finlay DK. Immunometabolism: cellular metabolism turns immune regulator. J Biol Chem. 2016;291:1-10.

3. Palsson-McDermott EM, Curtis AM, Goel G, Lauterbach MA, Sheedy FJ, Gleeson $L E$, et al. Pyruvate kinase $M 2$ regulates Hif-1alpha activity and IL-1 beta induction and is a critical determinant of the Warburg effect in LPS-activated macrophages. Cell Metab. 2015;21:65-80.

4. Moon JS, Hisata S, Park MA, DeNicola GM, Ryter SW, Nakahira K, et al. mTORC1induced HK1-dependent glycolysis regulates NLRP3 inflammasome activation. Cell Rep. 2015;12:102-15.

5. Chang $\mathrm{CH}$, Curtis JD, Maggi LB Jr, Faubert $B$, Villarino AV, O'Sullivan $D$, et al. Posttranscriptional control of $\mathrm{T}$ cell effector function by aerobic glycolysis. Cell. 2013;153:1239-51.

6. Michalek RD, Gerriets VA, Jacobs SR, Macintyre AN, Maclver NJ, Mason EF, et al. Cutting edge: distinct glycolytic and lipid oxidative metabolic programs are essential for effector and regulatory $\mathrm{CD} 4+\mathrm{T}$ cell subsets. J Immunol. 2011;186:3299-303.

7. Baardman J, Verberk S, Prange K, van Weeghel M, van der Velden S, Ryan DG, et al. A defective pentose phosphate pathway reduces inflammatory macrophage responses during hypercholesterolemia. Cell Rep. 2018;25:2044-52. e2045 
8. Rashida Gnanaprakasam JN, Wu R, Wang R. Metabolic reprogramming in modulating $\mathrm{T}$ cell reactive oxygen species generation and antioxidant capacity. Front Immunol. 2018;9:1075.

9. Krawczyk CM, Holowka T, Sun J, Blagih J, Amiel E, DeBerardinis RJ, et al. Toll-like receptor-induced changes in glycolytic metabolism regulate dendritic cell activation. Blood. 2010;115:4742-9.

10. Wang R, Dillon CP, Shi LZ, Milasta S, Carter R, Finkelstein D, et al. The transcription factor Myc controls metabolic reprogramming upon $T$ lymphocyte activation. Immunity. 2011;35:871-82.

11. Shi LZ, Wang R, Huang G, Vogel P, Neale G, Green DR, et al. HIF1alphadependent glycolytic pathway orchestrates a metabolic checkpoint for the differentiation of TH17 and Treg cells. J Exp Med. 2011;208:1367-76.

12. Saravia J, Raynor JL, Chapman NM, Lim SA, Chi H. Signaling networks in immunometabolism. Cell Res. 2020;30:328-42.

13. Huang $\mathrm{H}$, Long $\mathrm{L}$, Zhou $\mathrm{P}$, Chapman NM, Chi H. mTOR signaling at the crossroads of environmental signals and T-cell fate decisions. Immunol Rev. 2020;295:15-38.

14. Cheng SC, Quintin J, Cramer RA, Shepardson KM, Saeed S, Kumar V, et al. mTORand HIF-1alpha-mediated aerobic glycolysis as metabolic basis for trained immunity. Science. 2014;345:1250684.

15. Lee MKS, Al-Sharea A, Shihata WA, Bertuzzo Veiga C, Cooney OD, Fleetwood AJ, et al. Glycolysis is required for LPS-induced activation and adhesion of human CD14(+)CD16(-) monocytes. Front Immunol. 2019;10:2054.

16. Everts B, Amiel E, Huang SC, Smith AM, Chang CH, Lam WY, et al. TLR-driven early glycolytic reprogramming via the kinases TBK1-IKKvarepsilon supports the anabolic demands of dendritic cell activation. Nat Immunol. 2014;15:323-32.

17. Rodriguez-Espinosa O, Rojas-Espinosa O, Moreno-Altamirano MM, LopezVillegas EO, Sanchez-Garcia FJ. Metabolic requirements for neutrophil extracellular traps formation. Immunology. 2015;145:213-24.

18. Doughty CA, Bleiman BF, Wagner DJ, Dufort FJ, Mataraza JM, Roberts MF, et al. Antigen receptor-mediated changes in glucose metabolism in B lymphocytes: role of phosphatidylinositol 3-kinase signaling in the glycolytic control of growth. Blood. 2006;107:4458-65.

19. Donnelly RP, Loftus RM, Keating SE, Liou KT, Biron CA, Gardiner CM, et al. mTORC1-dependent metabolic reprogramming is a prerequisite for NK cell effector function. J Immunol. 2014;193:4477-84.

20. Lam WY, Becker AM, Kennerly KM, Wong R, Curtis JD, Llufrio EM, et al. Mitochondrial pyruvate import promotes long-term survival of antibody-secreting plasma cells. Immunity. 2016;45:60-73.

21. van der Windt GJ, Everts $B$, Chang $C H$, Curtis JD, Freitas TC, Amiel E, et al. Mitochondrial respiratory capacity is a critical regulator of $\mathrm{CD} 8+\mathrm{T}$ cell memory development. Immunity. 2012;36:68-78.

22. Mendoza A, Fang V, Chen C, Serasinghe M, Verma A, Muller J, et al. Lymphatic endothelial S1P promotes mitochondrial function and survival in naive $T$ cells. Nature. 2017;546:158-61.

23. Yang $K$, Shrestha $S$, Zeng $H$, Karmaus PW, Neale G, Vogel $P$, et al. T cell exit from quiescence and differentiation into Th2 cells depend on Raptor-mTORC1mediated metabolic reprogramming. Immunity. 2013;39:1043-56.

24. Wang F, Zhang S, Vuckovic I, Jeon R, Lerman A, Folmes CD, et al. Glycolytic stimulation is not a requirement for M2 macrophage differentiation. Cell Metab. 2018;28:463-75.e464.

25. Beier UH, Angelin A, Akimova T, Wang L, Liu Y, Xiao H, et al. Essential role of mitochondrial energy metabolism in Foxp3(+) T-regulatory cell function and allograft survival. FASEB J. 2015;29:2315-26.

26. Patsoukis N, Bardhan K, Chatterjee P, Sari D, Liu B, Bell LN, et al. PD-1 alters T-cell metabolic reprogramming by inhibiting glycolysis and promoting lipolysis and fatty acid oxidation. Nat Commun. 2015;6:6692

27. Jiang S. Mitochondrial oxidative phosphorylation is linked to T-cell exhaustion. Aging. 2020;12:16665-6.

28. Herzig S, Shaw RJ. AMPK: guardian of metabolism and mitochondrial homeostasis. Nat Rev Mol Cell Biol. 2018;19:121-35.

29. Gwinn DM, Shackelford DB, Egan DF, Mihaylova MM, Mery A, Vasquez DS, et al. AMPK phosphorylation of raptor mediates a metabolic checkpoint. Mol Cell. 2008;30:214-26.

30. Kishore M, Cheung K, Fu H, Bonacina F, Wang G, Coe D, et al. Regulatory T cell migration is dependent on glucokinase-mediated glycolysis. Immunity. 2017;47:875-89.e810.

31. Pacella I, Procaccini C, Focaccetti C, Miacci S, Timperi E, Faicchia D, et al. Fatty acid metabolism complements glycolysis in the selective regulatory $T$ cell expansion during tumor growth. Proc Natl Acad Sci USA. 2018;115:E6546-55.

32. De Rosa V, Galgani M, Porcellini A, Colamatteo A, Santopaolo M, Zuchegna C, et al. Glycolysis controls the induction of human regulatory $T$ cells by modulating the expression of FOXP3 exon 2 splicing variants. Nat Immunol. 2015;16:1174-84.
33. Saravia J, Zeng H, Dhungana Y, Bastardo Blanco D, Nguyen TM, Chapman NM, et al. Homeostasis and transitional activation of regulatory $T$ cells require c-Myc. Sci Adv. 2020;6:6443. eaaw6443

34. Raud B, Roy DG, Divakaruni AS, Tarasenko TN, Franke R, Ma EH, et al. Etomoxir actions on regulatory and memory $\mathrm{T}$ cells are independent of Cpt1a-mediated fatty acid oxidation. Cell Metab. 2018;28:504-15.e507.

35. Divakaruni AS, Hsieh WY, Minarrieta L, Duong TN, Kim K, Desousa BR, et al. Etomoxir inhibits macrophage polarization by disrupting $\mathrm{CoA}$ homeostasis. Cell Metab. 2018;28:490-503.e497.

36. Huang SC, Everts B, Ivanova Y, O'Sullivan D, Nascimento M, Smith AM, et al. Cellintrinsic lysosomal lipolysis is essential for alternative activation of macrophages. Nat Immunol. 2014;15:846-55.

37. Lampropoulou V, Sergushichev A, Bambouskova M, Nair S, Vincent EE, Loginicheva $E$, et al. Itaconate links inhibition of succinate dehydrogenase with macrophage metabolic remodeling and regulation of inflammation. Cell Metab. 2016;24:158-66.

38. Tannahill GM, Curtis AM, Adamik J, Palsson-McDermott EM, McGettrick AF, Goel $G$, et al. Succinate is an inflammatory signal that induces IL-1 beta through HIF1alpha. Nature. 2013;496:238-42.

39. Mills EL, Ryan DG, Prag HA, Dikovskaya D, Menon D, Zaslona Z, et al. Itaconate is an anti-inflammatory metabolite that activates Nrf2 via alkylation of KEAP1. Nature. 2018;556:113-7.

40. Arts RJ, Joosten LA, Netea MG. Immunometabolic circuits in trained immunity. Semin Immunol. 2016;28:425-30.

41. Ting JP, Lovering RC, Alnemri ES, Bertin J, Boss JM, Davis BK, et al. The NLR gene family: a standard nomenclature. Immunity. 2008;28:285-7.

42. Swanson KV, Deng M, Ting JP. The NLRP3 inflammasome: molecular activation and regulation to therapeutics. Nat Rev Immunol. 2019;19:477-89.

43. Schmid-Burgk JL, Chauhan D, Schmidt T, Ebert TS, Reinhardt J, Endl E, et al. A genome-wide CRISPR (clustered regularly interspaced short palindromic repeats) screen identifies NEK7 as an essential component of NLRP3 inflammasome activation. J Biol Chem. 2016;291:103-9.

44. He $Y$, Zeng MY, Yang D, Motro B, Nunez G. NEK7 is an essential mediator of NLRP3 activation downstream of potassium efflux. Nature. 2016;530:354-7.

45. Shi $H$, Wang $Y$, Li $X$, Zhan $X$, Tang $M$, Fina $M$, et al. NLRP3 activation and mitosis are mutually exclusive events coordinated by NEK7, a new inflammasome component. Nat Immunol. 2016;17:250-8.

46. Yang $Y$, Wang $H$, Kouadir $M$, Song $H$, Shi F. Recent advances in the mechanisms of NLRP3 inflammasome activation and its inhibitors. Cell Death Dis. 2019:10:128.

47. Juliana C, Fernandes-Alnemri T, Kang S, Farias A, Qin F, Alnemri ES. Nontranscriptional priming and deubiquitination regulate NLRP3 inflammasome activation. J Biol Chem. 2012;287:36617-22.

48. Py BF, Kim MS, Vakifahmetoglu-Norberg $H$, Yuan J. Deubiquitination of NLRP3 by BRCC3 critically regulates inflammasome activity. Mol Cell. 2013;49:331-8.

49. Song N, Liu ZS, Xue W, Bai ZF, Wang QY, Dai J, et al. NLRP3 phosphorylation is an essential priming event for inflammasome activation. Mol Cell. 2017;68:185-97. e186.

50. Barry R, John SW, Liccardi G, Tenev T, Jaco I, Chen $\mathrm{CH}$, et al. SUMO-mediated regulation of NLRP3 modulates inflammasome activity. Nat Commun. 2018;9:3001.

51. Segovia JA, Tsai SY, Chang TH, Shil NK, Weintraub ST, Short JD, et al. Nedd8 regulates inflammasome-dependent caspase-1 activation. Mol Cell Biol. 2015;35:582-97.

52. Bose S, Segovia JA, Somarajan SR, Chang T-H, Kannan TR, Baseman JB, et al. ADP-ribosylation of NLRP3 by Mycoplasma pneumoniae CARDS toxin regulates inflammasome activity. mBio. 2014;5:e02186-14.

53. He M, Chiang HH, Luo H, Zheng Z, Qiao Q, Wang L, et al. An acetylation switch of the NLRP3 inflammasome regulates aging-associated chronic inflammation and insulin resistance. Cell Metab. 2020;31:580-91.e585.

54. Yoon SJ, Park JY, Choi S, Lee JB, Jung H, Kim TD, et al. Ginsenoside Rg3 regulates S-nitrosylation of the NLRP3 inflammasome via suppression of iNOS. Biochem Biophys Res Commun. 2015;463:1184-9.

55. Bauernfeind FG, Horvath G, Stutz A, Alnemri ES, MacDonald K, Speert D, et al. Cutting edge: NF-kappaB activating pattern recognition and cytokine receptors license NLRP3 inflammasome activation by regulating NLRP3 expression. J Immunol. 2009;183:787-91.

56. Franchi L, Eigenbrod T, Nunez G. Cutting edge: TNF-alpha mediates sensitization to ATP and silica via the NLRP3 inflammasome in the absence of microbial stimulation. J Immunol. 2009:183:792-6.

57. Martinon F, Burns K, Tschopp J. The inflammasome: a molecular platform triggering activation of inflammatory caspases and processing of prolL-beta. Mol Cell. 2002;10:417-26. 
58. Kayagaki N, Stowe IB, Lee BL, O'Rourke K, Anderson K, Warming S, et al. Caspase11 cleaves gasdermin $D$ for non-canonical inflammasome signalling. Nature. 2015;526:666-71.

59. Liu X, Zhang Z, Ruan J, Pan Y, Magupalli VG, Wu H, et al. Inflammasomeactivated gasdermin $D$ causes pyroptosis by forming membrane pores. Nature. 2016;535:153-8.

60. Shi J, Zhao Y, Wang K, Shi X, Wang Y, Huang H, et al. Cleavage of GSDMD by inflammatory caspases determines pyroptotic cell death. Nature. 2015;526:660-5.

61. Liu Q, Zhang D, Hu D, Zhou X, Zhou Y. The role of mitochondria in NLRP3 inflammasome activation. Mol Immunol. 2018;103:115-24.

62. Wen H, Gris D, Lei Y, Jha S, Zhang L, Huang MT, et al. Fatty acid-induced NLRP3ASC inflammasome activation interferes with insulin signaling. Nat Immunol. 2011;12:408-15.

63. Vandanmagsar B, Youm YH, Ravussin A, Galgani JE, Stadler K, Mynatt RL, et al. The NLRP3 inflammasome instigates obesity-induced inflammation and insulin resistance. Nat Med. 2011;17:179-88.

64. Karasawa T, Kawashima A, Usui-Kawanishi F, Watanabe S, Kimura H, Kamata R, et al. Saturated fatty acids undergo intracellular crystallization and activate the NLRP3 inflammasome in macrophages. Arterioscler Thromb Vasc Biol. 2018;38:744-56.

65. Duewell $P$, Kono $H$, Rayner KJ, Sirois $C M$, Vladimer $G$, Bauernfeind FG, et al. NLRP3 inflammasomes are required for atherogenesis and activated by cholesterol crystals. Nature. 2010;464:1357-61.

66. Christ A, Günther $P$, Lauterbach $M$, Duewell $P$, Biswas D, Pelka K, et al. Western diet triggers NLRP3-dependent innate immune reprogramming. Cell. 2018;172:162-75.e114.

67. Stienstra R, Joosten LA, Koenen $T$, van Tits $B$, van Diepen JA, van den Berg SA, et al. The inflammasome-mediated caspase- 1 activation controls adipocyte differentiation and insulin sensitivity. Cell Metab. 2010;12:593-605.

68. Deng M, Guo H, Tam JW, Johnson BM, Brickey WJ, New JS, et al. Plateletactivating factor (PAF) mediates NLRP3-NEK7 inflammasome induction independently of PAFR. J Exp Med. 2019;216:2838-53.

69. Chalkiadaki A, Guarente L. High-fat diet triggers inflammation-induced cleavage of SIRT1 in adipose tissue to promote metabolic dysfunction. Cell Metab. 2012;16:180-8.

70. Camell CD, Günther P, Lee A, Goldberg EL, Spadaro O, Youm YH, et al. Aging induces an Nlrp3 inflammasome-dependent expansion of adipose $B$ cells that impairs metabolic homeostasis. Cell Metab. 2019;30:1024-39.e1026.

71. Masters SL, Dunne A, Subramanian SL, Hull RL, Tannahill GM, Sharp FA, et al. Activation of the NLRP3 inflammasome by islet amyloid polypeptide provides a mechanism for enhanced IL-1beta in type 2 diabetes. Nat Immunol. 2010;11:897-904.

72. van der Heijden T, Kritikou E, Venema W, van Duijn J, van Santbrink PJ, Slütter B, et al. NLRP3 inflammasome inhibition by MCC950 reduces atherosclerotic lesion development in apolipoprotein E-deficient mice-brief report. Arterioscler Thromb Vasc Biol. 2017;37:1457-61.

73. Ridker PM, Everett BM, Thuren T, MacFadyen JG, Chang WH, Ballantyne C, et al. Antiinflammatory therapy with canakinumab for atherosclerotic disease. $\mathrm{N}$ Engl J Med. 2017;377:1119-31.

74. Huang, $Y$, Jiang $H$, Chen $Y$, Wang $X$, Yang $Y$, Tao J, et al. Tranilast directly targets NLRP3 to treat inflammasome-driven diseases. EMBO Mol Med. 2018;10:e8689.

75. He H, Jiang H, Chen Y, Ye J, Wang A, Wang C, et al. Oridonin is a covalent NLRP3 inhibitor with strong anti-inflammasome activity. Nat Commun. 2018;9:2550.

76. Hooftman A, Angiari S, Hester S, Corcoran SE, Runtsch MC, Ling C, et al. The immunomodulatory metabolite itaconate modifies NLRP3 and inhibits inflammasome activation. Cell Metab. 2020;32:468-78.e467.

77. Youm YH, Nguyen KY, Grant RW, Goldberg EL, Bodogai M, Kim D, et al. The ketone metabolite beta-hydroxybutyrate blocks NLRP3 inflammasomemediated inflammatory disease. Nat Med. 2015;21:263-9.

78. Xie M, Yu Y, Kang R, Zhu S, Yang L, Zeng L, et al. PKM2-dependent glycolysis promotes NLRP3 and AIM2 inflammasome activation. Nat Commun. 2016;7:13280

79. Kang R, Tang D. PKR-dependent inflammatory signals. Sci Signal. 2012;5:pe47.

80. Lu B, Nakamura T, Inouye K, Li J, Tang Y, Lundbäck P, et al. Novel role of PKR in inflammasome activation and HMGB1 release. Nature. 2012;488:670-4.

81. Renaudin F, Orliaguet L, Castelli F, Fenaille F, Prignon A, Alzaid F, et al. Gout and pseudo-gout-related crystals promote GLUT1-mediated glycolysis that governs NLRP3 and interleukin-1beta activation on macrophages. Ann Rheum Dis. 2020;79:1506-14.

82. Coll RC, Robertson AA, Chae J, Higgins SC, Muñoz-Planillo R, Inserra MC, et al. A small-molecule inhibitor of the NLRP3 inflammasome for the treatment of inflammatory diseases. Nat Med. 2015;21:248-55.

83. Finucane OM, Sugrue J, Rubio-Araiz A, Guillot-Sestier MV, Lynch MA. The NLRP3 inflammasome modulates glycolysis by increasing PFKFB3 in an IL-1betadependent manner in macrophages. Sci Rep. 2019;9:4034.
84. Lin HC, Chen YJ, Wei YH, Lin HA, Chen CC, Liu TF, et al. Lactic acid fermentation is required for NLRP3 inflammasome activation. Front Immunol. 2021;12:630380.

85. Wolf AJ, Reyes CN, Liang W, Becker C, Shimada K, Wheeler ML, et al. Hexokinase is an innate immune receptor for the detection of bacterial peptidoglycan. Cell. 2016;166:624-36.

86. Shimada K, Crother TR, Karlin J, Dagvadorj J, Chiba N, Chen S, et al. Oxidized mitochondrial DNA activates the NLRP3 inflammasome during apoptosis. Immunity. 2012;36:401-14.

87. Zhong Z, Umemura A, Sanchez-Lopez E, Liang S, Shalapour S, Wong J, et al. NFkappaB restricts inflammasome activation via elimination of damaged mitochondria. Cell. 2016;164:896-910.

88. Nakahira K, Haspel JA, Rathinam VA, Lee SJ, Dolinay T, Lam HC, et al. Autophagy proteins regulate innate immune responses by inhibiting the release of mitochondrial DNA mediated by the NALP3 inflammasome. Nat Immunol. 2011;12:222-30.

89. Sanman LE, Qian Y, Eisele NA, Ng TM, van der Linden WA, Monack DM, et al. Disruption of glycolytic flux is a signal for inflammasome signaling and pyroptotic cell death. Elife. 2016;5:e13663.

90. Anastasiou D, Yu Y, Israelsen WJ, Jiang JK, Boxer MB, Hong BS, et al. Pyruvate kinase $\mathrm{M} 2$ activators promote tetramer formation and suppress tumorigenesis. Nat Chem Biol. 2012;8:839-47.

91. Luo W, Hu H, Chang R, Zhong J, Knabel M, O'Meally R, et al. Pyruvate kinase M2 is a PHD3-stimulated coactivator for hypoxia-inducible factor 1. Cell. 2011;145:732-44.

92. Yang W, Lu Z. Nuclear PKM2 regulates the Warburg effect. Cell Cycle. 2013;12:3154-8.

93. Mills $E, O^{\prime}$ Neill LA. Succinate: a metabolic signal in inflammation. Trends Cell Biol. 2014;24:313-20.

94. Selak MA, Armour SM, MacKenzie ED, Boulahbel H, Watson DG, Mansfield KD, et al. Succinate links TCA cycle dysfunction to oncogenesis by inhibiting HIFalpha prolyl hydroxylase. Cancer Cell. 2005;7:77-85.

95. Rubic T, Lametschwandtner G, Jost S, Hinteregger S, Kund J, Carballido-Perrig N, et al. Triggering the succinate receptor GPR91 on dendritic cells enhances immunity. Nat Immunol. 2008;9:1261-9.

96. He W, Miao FJ, Lin DC, Schwandner RT, Wang Z, Gao J, et al. Citric acid cycle intermediates as ligands for orphan G-protein-coupled receptors. Nature. 2004;429:188-93.

97. Bruick RK, McKnight SL. A conserved family of prolyl-4-hydroxylases that modify HIF. Science. 2001;294:1337-40.

98. Minchenko A, Leshchinsky I, Opentanova I, Sang N, Srinivas V, Armstead V, et al. Hypoxia-inducible factor-1-mediated expression of the 6-phosphofructo-2kinase/fructose-2,6-bisphosphatase-3 (PFKFB3) gene. Its possible role in the Warburg effect. J Biol Chem. 2002;277:6183-7.

99. Bambouskova M, Potuckova L, Paulenda T, Kerndl M, Mogilenko DA, Lizotte K, et al. Itaconate confers tolerance to late NLRP3 inflammasome activation. Cell Rep. 2021;34:108756.

100. Cordes T, Wallace M, Michelucci A, Divakaruni AS, Sapcariu SC, Sousa C, et al. Immunoresponsive gene 1 and itaconate inhibit succinate dehydrogenase to modulate intracellular succinate levels. J Biol Chem. 2016;291:14274-84.

101. Liao ST, Han C, Xu DQ, Fu XW, Wang JS, Kong LY. 4-Octyl itaconate inhibits aerobic glycolysis by targeting GAPDH to exert anti-inflammatory effects. Nat Commun. 2019;10:5091.

102. Qin W, Qin K, Zhang Y, Jia W, Chen Y, Cheng B, et al. S-glycosylation-based cysteine profiling reveals regulation of glycolysis by itaconate. Nat Chem Biol. 2019;15:983-91.

103. Li S, Liang F, Kwan K, Tang Y, Wang X, Tang $Y$, et al. Identification of ethyl pyruvate as a NLRP3 inflammasome inhibitor that preserves mitochondrial integrity. Mol Med. 2018;24:8.

104. Zhong $X$, Xie L, Yang X, Liang F, Yang Y, Tong J, et al. Ethyl pyruvate protects against sepsis-associated encephalopathy through inhibiting the NLRP3 inflammasome. Mol Med. 2020;26:55.

105. Liu X, Zhou W, Zhang X, Lu P, Du Q, Tao L, et al. Dimethyl fumarate ameliorates dextran sulfate sodium-induced murine experimental colitis by activating Nrf2 and suppressing NLRP3 inflammasome activation. Biochem Pharm. 2016;112:37-49.

106. Subramanian N, Natarajan K, Clatworthy MR, Wang Z, Germain RN. The adaptor MAVS promotes NLRP3 mitochondrial localization and inflammasome activation. Cell. 2013;153:348-61.

107. Zhou R, Yazdi AS, Menu P, Tschopp J. A role for mitochondria in NLRP3 inflammasome activation. Nature. 2011;469:221-5.

108. Li X, Kazgan N. Mammalian sirtuins and energy metabolism. Int J Biol Sci. 2011;7:575-87.

109. Misawa T, Takahama M, Kozaki T, Lee H, Zou J, Saitoh T, et al. Microtubule-driven spatial arrangement of mitochondria promotes activation of the NLRP3 inflammasome. Nat Immunol. 2013;14:454-60. 
110. Cameron AM, Castoldi A, Sanin DE, Flachsmann LJ, Field CS, Puleston DJ, et al. Inflammatory macrophage dependence on $\mathrm{NAD}(+)$ salvage is a consequence of reactive oxygen species-mediated DNA damage. Nat Immunol. 2019;20:420-32.

111. Molla MD, Ayelign B, Dessie G, Geto Z, Admasu TD. Caspase-1 as a regulatory molecule of lipid metabolism. Lipids Health Dis. 2020;19:34.

112. Meyers, AK \& Zhu, X. The NLRP3 inflammasome: metabolic regulation and contribution to inflammaging. Cells 2020;9:1808.

113. Yan $Y$, Jiang W, Spinetti T, Tardivel A, Castillo R, Bourquin C, et al. Omega-3 fatty acids prevent inflammation and metabolic disorder through inhibition of NLRP3 inflammasome activation. Immunity. 2013;38:1154-63.

114. Shen L, Yang Y, Ou T, Key CC, Tong SH, Sequeira RC, et al. Dietary PUFAs attenuate NLRP3 inflammasome activation via enhancing macrophage autophagy. J Lipid Res. 2017;58:1808-21.

115. Goldberg EL, Asher JL, Molony RD, Shaw AC, Zeiss CJ, Wang C, et al. betaHydroxybutyrate Deactivates Neutrophil NLRP3 Inflammasome to Relieve Gout Flares. Cell Rep. 2017;18:2077-87.

116. Moon JS, Nakahira K, Chung KP, DeNicola GM, Koo MJ, Pabón MA, et al. NOX4dependent fatty acid oxidation promotes NLRP3 inflammasome activation in macrophages. Nat Med. 2016;22:1002-12.

117. Qin YY, Li M, Feng X, Wang J, Cao L, Shen XK, et al. Combined NADPH and the NOX inhibitor apocynin provides greater anti-inflammatory and neuroprotective effects in a mouse model of stroke. Free Radic Biol Med. 2017;104:333-45.

118. Arnoult D, Soares F, Tattoli I, Castanier C, Philpott DJ, Girardin SE. An N-terminal addressing sequence targets NLRX1 to the mitochondrial matrix. J Cell Sci. 2009;122:3161-8.

119. Moore CB, Bergstralh DT, Duncan JA, Lei Y, Morrison TE, Zimmermann AG, et al. NLRX1 is a regulator of mitochondrial antiviral immunity. Nature. 2008;451:573-7.

120. Tattoli I, Carneiro LA, Jéhanno M, Magalhaes JG, Shu Y, Philpott DJ, et al. NLRX1 is a mitochondrial NOD-like receptor that amplifies NF-kappaB and JNK pathways by inducing reactive oxygen species production. EMBO Rep. 2008;9:293-300.

121. Feng $H$, Lenarcic EM, Yamane $D$, Wauthier $E, M o J$, Guo $H$, et al. NLRX1 promotes immediate IRF1-directed antiviral responses by limiting dsRNA-activated translational inhibition mediated by PKR. Nat Immunol. 2017;18:1299-309.

122. Hong $\mathrm{M}$, Yoon $\mathrm{SI}$, Wilson IA. Structure and functional characterization of the RNA-binding element of the NLRX1 innate immune modulator. Immunity. 2012;36:337-47.

123. Li X, Deng M, Petrucelli AS, Zhu C, Mo J, Zhang L, et al. Viral DNA binding to NLRC3, an inhibitory nucleic acid sensor, unleashes STING, a cyclic dinucleotide receptor that activates type I interferon. Immunity. 2019;50:591-9.e596.

124. Xia X, Cui J, Wang HY, Zhu L, Matsueda S, Wang Q, et al. NLRX1 negatively regulates TLR-induced NF-kappaB signaling by targeting TRAF6 and IKK. Immunity. 2011;34:843-53.

125. Allen IC, Moore CB, Schneider M, Lei Y, Davis BK, Scull MA, et al. NLRX1 protein attenuates inflammatory responses to infection by interfering with the RIG-IMAVS and TRAF6-NF-kappaB signaling pathways. Immunity. 2011;34:854-65.

126. Guo H, König R, Deng M, Riess M, Mo J, Zhang L, et al. NLRX1 sequesters STING to negatively regulate the interferon response, thereby facilitating the replication of HIV-1 and DNA viruses. Cell Host Microbe. 2016;19:515-28.

127. Kastelberg B, Tubau-Juni N, Ayubi T, Leung A, Leber A, Hontecillas R, et al. NLRX1 is a key regulator of immune signaling during invasive pulmonary aspergillosis. PLoS Pathog. 2020;16:e1008854.

128. Philipson CW, Bassaganya-Riera J, Viladomiu M, Kronsteiner B, Abedi V, Hoops S, et al. Modeling the regulatory mechanisms by which NLRX1 modulates innate immune responses to Helicobacter pylori infection. PLoS ONE. 2015;10:e0137839.

129. Gordon DE, Jang GM, Bouhaddou M, Xu J, Obernier K, White KM, et al. A SARS CoV-2 protein interaction map reveals targets for drug repurposing. Nature. 2020;583:459-68.

130. Zhang Y, Yao Y, Qiu X, Wang G, Hu Z, Chen S, et al. Listeria hijacks host mitophagy through a novel mitophagy receptor to evade killing. Nat Immunol. 2019;20:433-46.

131. Huang JH, Liu CY, Wu SY, Chen WY, Chang TH, Kan HW, et al. NLRX1 facilitates histoplasma capsulatum-induced LC3-associated phagocytosis for cytokine production in macrophages. Front Immunol. 2018;9:2761.

132. Lei $Y$, Wen $H$, Ting JP. The NLR protein, NLRX1, and its partner, TUFM, reduce type I interferon, and enhance autophagy. Autophagy. 2013;9:432-3.

133. Lei $Y$, Wen H, Yu Y, Taxman DJ, Zhang L, Widman DG, et al. The mitochondrial proteins NLRX1 and TUFM form a complex that regulates type I interferon and autophagy. Immunity. 2012;36:933-46.

134. Theus MH, Brickler T, Meza AL, Coutermarsh-Ott S, Hazy A, Gris D, et al. Loss of NLRX1 exacerbates neural tissue damage and NF-kappaB signaling following brain injury. J Immunol. 2017;199:3547-58.

135. Kang MJ, Yoon CM, Kim BH, Lee CM, Zhou Y, Sauler M, et al. Suppression of NLRX1 in chronic obstructive pulmonary disease. J Clin Invest. 2015;125:2458-62.
136. Jang $\mathrm{H}$, Kim M, Hong JY, Cho HJ, Kim CH, Kim YH, et al. Mitochondrial and nuclear mitochondrial variants in allergic diseases. Allergy Asthma Immunol Res. 2020;12:877-84.

137. Kim HW, Kwon YJ, Park BW, Song JJ, Park YB, Park MC. Differential expressions of NOD-like receptors and their associations with inflammatory responses in rheumatoid arthritis. Clin Exp Rheumatol. 2017;35:630-7.

138. Eitas TK, Chou WC, Wen H, Gris D, Robbins GR, Brickey J, et al. The nucleotidebinding leucine-rich repeat (NLR) family member NLRX1 mediates protection against experimental autoimmune encephalomyelitis and represses macrophage/microglia-induced inflammation. J Biol Chem. 2014;289:4173-9.

139. Gharagozloo M, Mahmoud S, Simard C, Yamamoto K, Bobbala D, llangumaran S, et al. NLRX1 inhibits the early stages of CNS inflammation and prevents the onset of spontaneous autoimmunity. PLoS Biol. 2019;17:e3000451.

140. Tattoli I, Killackey SA, Foerster EG, Molinaro R, Maisonneuve C, Rahman MA, et al. NLRX1 acts as an epithelial-intrinsic tumor suppressor through the modulation of TNF-mediated proliferation. Cell Rep. 2016;14:2576-86.

141. Lei A, Maloy KJ. Colon cancer in the land of NOD: NLRX1 as an intrinsic tumor suppressor. Trends Immunol. 2016;37:569-70.

142. Koblansky AA, Truax AD, Liu R, Montgomery SA, Ding S, Wilson JE, et al. The innate immune receptor NLRX1 functions as a tumor suppressor by reducing colon tumorigenesis and key tumor-promoting signals. Cell Rep. 2016;14:2562-75.

143. Coutermarsh-Ott S, Simmons A, Capria V, LeRoith T, Wilson JE, Heid B, et al. NLRX1 suppresses tumorigenesis and attenuates histiocytic sarcoma through the negative regulation of NF-kappaB signaling. Oncotarget. 2016;7:33096-110.

144. Singh K, Poteryakhina A, Zheltukhin A, Bhatelia K, Prajapati P, Sripada L, et al. NLRX1 acts as tumor suppressor by regulating TNF-alpha induced apoptosis and metabolism in cancer cells. Biochim Biophys Acta. 2015;1853:1073-86.

145. Pickering RJ, Booty LM. NLR in eXile: emerging roles of NLRX1 in immunity and human disease. Immunology. 2021;162:268-80.

146. Nagai-Singer MA, Morrison HA, Allen IC. NLRX1 is a multifaceted and enigmatic regulator of immune system function. Front Immunol. 2019;10:2419.

147. Soares F, Tattoli I, Rahman MA, Robertson SJ, Belcheva A, Liu D, et al. The mitochondrial protein NLRX1 controls the balance between extrinsic and intrinsic apoptosis. J Biol Chem. 2014;289:19317-30.

148. Singh K, Roy M, Prajapati P, Lipatova A, Sripada L, Gohel D, et al. NLRX1 regulates TNF-alpha-induced mitochondria-lysosomal crosstalk to maintain the invasive and metastatic potential of breast cancer cells. Biochim Biophys Acta Mol Basis Dis. 2019;1865:1460-76.

149. Lu P, Hontecillas R, Abedi V, Kale S, Leber A, Heltzel C, et al. Modeling-enabled characterization of novel NLRX1 ligands. PLoS ONE. 2015;10:e0145420.

150. Stokman G, Kors L, Bakker PJ, Rampanelli E, Claessen N, Teske G, et al. NLRX1 dampens oxidative stress and apoptosis in tissue injury via control of mitochondrial activity. J Exp Med. 2017;214:2405-20.

151. Plaitakis, A, Kalef-Ezra, E, Kotzamani, D, Zaganas, I \& Spanaki, C The glutamate dehydrogenase pathway and its roles in cell and tissue biology in health and disease. Biology. 2017;6:11.

152. Leber A, Hontecillas R, Tubau-Juni N, Zoccoli-Rodriguez V, Abedi V, BassaganyaRiera J. NLRX1 modulates immunometabolic mechanisms controlling the hostgut microbiota interactions during inflammatory bowel disease. Front Immunol. 2018;9:363.

153. Leber A, Hontecillas R, Tubau-Juni N, Zoccoli-Rodriguez V, Hulver M, McMillan R, et al. NLRX1 regulates effector and metabolic functions of CD4(+) T cells. J Immunol. 2017;198:2260-8.

154. Leber, A et al. Exploratory studies with NX-13: oral toxicity and pharmacokinetics in rodents of an orally active, gut-restricted first-in-class therapeutic for IBD that targets NLRX1. Drug Chem Toxicol. 1-6 (2019).

155. Leber A, Hontecillas R, Zoccoli-Rodriguez V, Bienert C, Chauhan J, BassaganyaRiera J. Activation of NLRX1 by NX-13 alleviates inflammatory bowel disease through immunometabolic mechanisms in $\mathrm{CD4}(+) \mathrm{T}$ cells. J Immunol. 2019;203:3407-15

156. Kors L, Rampanelli E, Stokman G, Butter LM, Held NM, Claessen N, et al. Deletion of NLRX1 increases fatty acid metabolism and prevents diet-induced hepatic steatosis and metabolic syndrome. Biochim Biophys Acta Mol Basis Dis. 2018;1864:1883-95.

157. Costford SR, Tattoli I, Duan FT, Volchuk A, Klip A, Philpott DJ, et al. Male mice lacking NLRX1 are partially protected from high-fat diet-induced hyperglycemia. J Endocr Soc. 2018;2:336-47.

158. Jourdain AA, Koppen $M$, Rodley CD, Maundrell K, Gueguen N, Reynier $P$, et al. A mitochondria-specific isoform of FASTK is present in mitochondrial RNA granules and regulates gene expression and function. Cell Rep. 2015;10:1110-21.

159. Li S, Wang L, Berman M, Kong YY, Dorf ME. Mapping a dynamic innate immunity protein interaction network regulating type I interferon production. Immunity. 2011;35:426-40. 
160. Singh K, Sripada L, Lipatova A, Roy M, Prajapati P, Gohel D, et al. NLRX1 resides in mitochondrial RNA granules and regulates mitochondrial RNA processing and bioenergetic adaptation. Biochim Biophys Acta Mol Cell Res. 2018;1865:1260-76.

161. Guo H, Wang Q, Ghneim K, Wang L, Rampanelli E, Holley-Guthrie E, et al. Multiomics analyses reveal that HIV-1 alters CD4(+) T cell immunometabolism to fuel virus replication. Nat Immunol. 2021;22:423-33.

162. Constantinescu CS, Faroogi N, O'Brien K, Gran B. Experimental autoimmune encephalomyelitis (EAE) as a model for multiple sclerosis (MS). $\mathrm{Br} J$ Pharm. 2011;164:1079-106.

163. Lim S, Kim WJ, Kim YH, Lee S, Koo JH, Lee JA, et al. dNP2 is a blood-brain barrierpermeable peptide enabling ctCTLA-4 protein delivery to ameliorate experimental autoimmune encephalomyelitis. Nat Commun. 2015;6:8244.

164. Koo JH, Kim DH, Cha D, Kang MJ, Choi JM. LRR domain of NLRX1 protein delivery by dNP2 inhibits T cell functions and alleviates autoimmune encephalomyelitis. Theranostics. 2020;10:3138-50.

165. Harton JA, Linhoff MW, Zhang J, Ting JP. Cutting edge: CATERPILLER: a large family of mammalian genes containing CARD, pyrin, nucleotide-binding, and leucine-rich repeat domains. J Immunol. 2002;169:4088-93.

166. Conti BJ, Davis BK, Zhang J, O,connor W Jr, Williams KL, Ting JP. CATERPILLER 16.2 (CLR16.2), a novel NBD/LRR family member that negatively regulates $T$ cell function. J Biol Chem. 2005;280:18375-85.

167. Schneider M, Zimmermann AG, Roberts RA, Zhang L, Swanson KV, Wen H, et al. The innate immune sensor NLRC3 attenuates Toll-like receptor signaling via modification of the signaling adaptor TRAF6 and transcription factor NF-kappaB. Nat Immunol. 2012;13:823-31.

168. Zhang L, Mo J, Swanson KV, Wen H, Petrucelli A, Gregory SM, et al. NLRC3, a member of the NLR family of proteins, is a negative regulator of innate immune signaling induced by the DNA sensor STING. Immunity. 2014;40:329-41.

169. Tocker AM, Durocher E, Jacob KD, Trieschman KE, Talento SM, Rechnitzer AA, et al. The scaffolding protein IQGAP1 interacts with NLRC3 and inhibits type I IFN production. J Immunol. 2017;199:2896-909.

170. Karki R, Man SM, Malireddi R, Kesavardhana S, Zhu Q, Burton AR, et al. NLRC3 is an inhibitory sensor of PI3K-mTOR pathways in cancer. Nature. 2016;540:583-7.

171. Karki R, Malireddi RKS, Zhu Q, Kanneganti TD. NLRC3 regulates cellular proliferation and apoptosis to attenuate the development of colorectal cancer. Cell Cycle. 2017;16:1243-51.

172. Zeng $\mathrm{H}$, Chi H. mTOR signaling in the differentiation and function of regulatory and effector T cells. Curr Opin Immunol. 2017:46:103-11.

173. Fruman DA, Chiu H, Hopkins BD, Bagrodia S, Cantley LC, Abraham RT. The PI3K pathway in human disease. Cell. 2017;170:605-35.

174. Saxton RA, Sabatini DM. mTOR signaling in growth, metabolism, and disease. Cell. 2017;169:361-71.

175. Kim J, Guan KL. mTOR as a central hub of nutrient signalling and cell growth. Nat Cell Biol. 2019;21:63-71.

176. Hu S, Du X, Huang Y, Fu Y, Yang Y, Zhan X, et al. NLRC3 negatively regulates $\mathrm{CD} 4+\mathrm{T}$ cells and impacts protective immunity during Mycobacterium tuberculosis infection. PLoS Pathog. 2018;14:e1007266.

177. Uchimura T, Oyama Y, Deng M, Guo H, Wilson JE, Rampanelli E, et al. The innate immune sensor NLRC3 acts as a rheostat that fine-tunes T Cell responses in infection and autoimmunity. Immunity. 2018;49:1049-61.e1046.

178. Zha LH, Zhou J, Tan Y, Guo S, Zhang MQ, Li S, et al. NLRC3 inhibits PDGFinduced PASMCs proliferation via PI3K-mTOR pathway. J Cell Physiol. 2020;235:9557-67.

179. Rathinam VA, Jiang Z, Waggoner SN, Sharma S, Cole LE, Waggoner L, et al. The AIM2 inflammasome is essential for host defense against cytosolic bacteria and DNA viruses. Nat Immunol. 2010;11:395-402.

180. Krieg AM. AIMing 2 defend against intracellular pathogens. Nat Immunol. 2010;11:367-9.

181. Fernandes-Alnemri T, Yu JW, Juliana C, Solorzano L, Kang S, Wu J, et al. The AIM2 inflammasome is critical for innate immunity to Francisella tularensis. Nat Immunol. 2010;11:385-93.

182. Fernandes-Alnemri T, Yu JW, Datta P, Wu J, Alnemri ES. AIM2 activates the inflammasome and cell death in response to cytoplasmic DNA. Nature. 2009;458:509-13.

183. Rathinam VA, Vanaja SK, Fitzgerald KA. Regulation of inflammasome signaling. Nat Immunol. 2012;13:333-42.

184. Jin T, Perry A, Jiang J, Smith P, Curry JA, Unterholzner L, et al. Structures of the HIN domain:DNA complexes reveal ligand binding and activation mechanisms of the AIM2 inflammasome and IFI16 receptor. Immunity. 2012;36:561-71.

185. Jin T, Perry A, Smith $P$, Jiang J, Xiao TS. Structure of the absent in melanoma 2 (AIM2) pyrin domain provides insights into the mechanisms of AIM2 autoinhibition and inflammasome assembly. J Biol Chem. 2013;288:13225-35.

186. Kumari $P$, Russo AJ, Shivcharan S, Rathinam VA. AIM2 in health and disease: Inflammasome and beyond. Immunol Rev. 2020:297:83-95.
187. Sharma BR, Karki R, Kanneganti TD. Role of AIM2 inflammasome in inflammatory diseases, cancer and infection. Eur J Immunol. 2019;49:1998-2011.

188. Man SM, Karki R, Kanneganti TD. AIM2 inflammasome in infection, cancer, and autoimmunity: Role in DNA sensing, inflammation, and innate immunity. Eur J Immunol. 2016;46:269-80.

189. Hotchkiss RS, Moldawer LL, Opal SM, Reinhart K, Turnbull IR, Vincent JL. Sepsis and septic shock. Nat Rev Dis Prim. 2016;2:16045.

190. Yang L, Xie M, Yang M, Yu Y, Zhu S, Hou W, et al. PKM2 regulates the Warburg effect and promotes HMGB1 release in sepsis. Nat Commun. 2014;5:4436.

191. Cho SJ, Moon JS, Nikahira K, Yun HS, Harris R, Hong KS, et al. GLUT1-dependent glycolysis regulates exacerbation of fibrosis via AIM2 inflammasome activation. Thorax. 2020;75:227-36.

192. DeYoung KL, Ray ME, Su YA, Anzick SL, Johnstone RW, Trapani JA, et al. Cloning a novel member of the human interferon-inducible gene family associated with control of tumorigenicity in a model of human melanoma. Oncogene. 1997;15:453-7.

193. Dihlmann S, Tao S, Echterdiek F, Herpel E, Jansen L, Chang-Claude J, et al. Lack of Absent in Melanoma 2 (AIM2) expression in tumor cells is closely associated with poor survival in colorectal cancer patients. Int J Cancer. 2014;135:2387-96.

194. Kim TM, Laird PW, Park PJ. The landscape of microsatellite instability in colorectal and endometrial cancer genomes. Cell. 2013;155:858-68.

195. Woerner SM, Kloor M, Schwitalle Y, Youmans H, Doeberitz MV, Gebert J, et al. The putative tumor suppressor AIM2 is frequently affected by different genetic alterations in microsatellite unstable colon cancers. Genes Chromosomes Cancer. 2007;46:1080-9.

196. Wilson, JE, Petrucelli A S, Chen L, Koblansky A A, Truax A D, Oyama Y, et al. Inflammasome-independent role of AIM2 in suppressing colon tumorigenesis via DNA-PK and Akt. Nat Med. 2015;21:906-13.

197. Man SM, Zhu Q, Zhu L, Liu Z, Karki R, Malik A, et al. Critical role for the DNA sensor AIM2 in stem. Cell Prolif Cancer Cell. 2015;162:45-58.

198. Ma X, Guo P, Qiu Y, Mu K, Zhu L, Zhao W, et al. Loss of AIM2 expression promotes hepatocarcinoma progression through activation of mTOR-S6K1 pathway. Oncotarget. 2016;7:36185-97.

199. Chen SL, Liu LL, Lu SX, Luo RZ, Wang CH, Wang H, et al. HBx-mediated decrease of AIM2 contributes to hepatocellular carcinoma metastasis. Mol Oncol. 2017;11:1225-40.

200. Xu F, Na L, Li Y, Chen L. Roles of the PI3K/AKT/mTOR signalling pathways in neurodegenerative diseases and tumours. Cell Biosci. 2020;10:54.

201. Ma, C, Li S, Hu Y, Ma Y, Wu Y, Wu C, et al. AIM2 controls microglial inflammation to prevent experimental autoimmune encephalomyelitis. J Exp Med. 2021;218:e20201796.

202. Inoue M, Chen PH, Siecinski S, Li QJ, Liu C, Steinman L, et al. An interferon-betaresistant and NLRP3 inflammasome-independent subtype of EAE with neuronal damage. Nat Neurosci. 2016;19:1599-609.

203. Chou WC, Guo Z, Guo H, Chen L, Zhang G, Liang K, et al. AIM2 in regulatory T cells restrains autoimmune diseases. Nature. 2021;591:300-5.

204. Martin BN, Wang C, Zhang CJ, Kang Z, Gulen MF, Zepp JA, et al. T cell-intrinsic ASC critically promotes $T(H) 17$-mediated experimental autoimmune encephalomyelitis. Nat Immunol. 2016;17:583-92.

205. Shaw PJ, Lukens JR, Burns S, Chi H, McGargill MA, Kanneganti TD. Cutting edge: critical role for PYCARD/ASC in the development of experimental autoimmune encephalomyelitis. J Immunol. 2010;184:4610-4.

206. Gris $D$, Ye $Z$, locca $H A$, Wen $H$, Craven RR, Gris $P$, et al. NLRP3 plays a critical role in the development of experimental autoimmune encephalomyelitis by mediating Th1 and Th17 responses. J Immunol. 2010;185:974-81.

207. Furlan R, Martino G, Galbiati F, Poliani PL, Smiroldo S, Bergami A, et al. Caspase-1 regulates the inflammatory process leading to autoimmune demyelination. J Immunol. 1999;163:2403-9.

208. Jin L, Waterman PM, Jonscher KR, Short CM, Reisdorph NA, Cambier JC. MPYS, a novel membrane tetraspanner, is associated with major histocompatibility complex class II and mediates transduction of apoptotic signals. Mol Cell Biol. 2008;28:5014-26.

209. Zhong B, Yang Y, Li S, Wang YY, Li Y, Diao F, et al. The adaptor protein MITA links virus-sensing receptors to IRF3 transcription factor activation. Immunity. 2008;29:538-50.

210. Ishikawa H, Ma Z, Barber GN. STING regulates intracellular DNA-mediated, type I interferon-dependent innate immunity. Nature. 2009;461:788-92.

211. Sun W, Li Y, Chen $L$, Chen H, You F, Zhou X, et al. ERIS, an endoplasmic reticulum IFN stimulator, activates innate immune signaling through dimerization. Proc Natl Acad Sci USA. 2009;106:8653-8.

212. Cao DJ, Schiattarella GG, Villalobos E, Jiang N, May HI, Li T, et al. Cytosolic DNA sensing promotes macrophage transformation and governs myocardial ischemic injury. Circulation. 2018;137:2613-34.

213. Downey CM, Aghaei M, Schwendener RA, Jirik FR. DMXAA causes tumor sitespecific vascular disruption in murine non-small cell lung cancer, and like the 
endogenous non-canonical cyclic dinucleotide STING agonist, 2'3'-cGAMP, induces M2 macrophage repolarization. PLoS ONE. 2014;9:e99988.

214. Cheng, N, Watkins-Schulz R, Junkins R D, David C N, Johnson B M, Montgomery $S \mathrm{~A}$, et al. A nanoparticle-incorporated STING activator enhances antitumor immunity in PD-L1-insensitive models of triple-negative breast cancer. $\mathrm{JCl}$ Insight. 2018;3:e120638.

215. Olson GS, Murray TA, Jahn AN, Mai D, Diercks AH, Gold ES, et al. Type I interferon decreases macrophage energy metabolism during mycobacterial infection. Cell Rep. 2021;35:109195.

216. Wang F, Alain T, Szretter KJ, Stephenson K, Pol JG, Atherton MJ, et al. S6K-STING interaction regulates cytosolic DNA-mediated activation of the transcription factor IRF3. Nat Immunol. 2016;17:514-22.

217. Meade N, Furey C, Li H, Verma R, Chai Q, Rollins MG, et al. Poxviruses evade cytosolic sensing through disruption of an mTORC1-mTORC2 regulatory circuit. Cell. 2018;174:1143-57.e1117.

218. Jing, Y, D Xin, Y Lu, K Danqing, Jiang P, Li N, Cheng J, et al. STING couples with PI3K to regulate actin reorganization during BCR activation. Sci Adv. 2020;6:eaax9455.

219. Larkin B, llyukha V, Sorokin M, Buzdin A, Vannier E, Poltorak A. Cutting Edge: activation of STING in T cells induces type I IFN responses and cell death. J Immunol. 2017;199:397-402.

220. Imanishi, T, Unno M, Kobayashi W, Yoneda N, Matsuda S, Ikeda K, et al. Reciprocal regulation of STING and TCR signaling by $\mathrm{mTORC} 1$ for T-cell activation and function. Life Sci Alliance. 2019;2:e201800282.

221. Jia M, Qin D, Zhao C, Chai L, Yu Z, Wang W, et al. Redox homeostasis maintained by GPX4 facilitates STING activation. Nat Immunol. 2020;21:727-35.

222. Olagnier D, Brandtoft AM, Gunderstofte C, Villadsen NL, Krapp C, Thielke AL et al. Nrf2 negatively regulates STING indicating a link between antiviral sensing and metabolic reprogramming. Nat Commun. 2018;9:3506.

223. Hansen AL, Buchan GJ, Rühl M, Mukai K, Salvatore SR, Ogawa E, et al. Nitro-fatty acids are formed in response to virus infection and are potent inhibitors of STING palmitoylation and signaling. Proc Natl Acad Sci USA. 2018;115:E7768-75.

224. Hsu SC, Chen CL, Cheng ML, Chu CY, Changou CA, Yu YL, et al. Arginine starvation elicits chromatin leakage and CGAS-STING activation via epigenetic silencing of metabolic and DNA-repair genes. Theranostics. 2021;11:7527-45.

225. Bai J, Liu F. The cGAS-cGAMP-STING pathway: a molecular link between immunity and metabolism. Diabetes. 2019;68:1099-108.

226. Chen, C, Yang, RX \& Xu, HG. STING and liver disease. J Gastroenterol. 2021;56:704-12.

227. Luo X, Li H, Ma L, Zhou J, Guo X, Woo SL, et al. Expression of STING is increased in liver tissues from patients with NAFLD and promotes macrophage-mediated hepatic inflammation and fibrosis in mice. Gastroenterology. 2018;155:1971-84. e1974.

228. Yu Y, Liu Y, An W, Song J, Zhang Y, Zhao X. STING-mediated inflammation in Kupffer cells contributes to progression of nonalcoholic steatohepatitis. J Clin Invest. 2019;129:546-55.

229. Bai J, Cervantes C, He S, He J, Plasko GR, Wen J, et al. Mitochondrial stressactivated CGAS-STING pathway inhibits thermogenic program and contributes to overnutrition-induced obesity in mice. Commun Biol. 2020;3:257.
230. Bai J, Cervantes C, Liu J, He S, Zhou H, Zhang B, et al. DsbA-L prevents obesityinduced inflammation and insulin resistance by suppressing the mtDNA releaseactivated CGAS-cGAMP-STING pathway. Proc Natl Acad Sci USA. 2017;114:12196-201.

231. Palsson-McDermott EM, O'Neill LAJ. Targeting immunometabolism as an antiinflammatory strategy. Cell Res. 2020;30:300-14.

232. Artyomov MN, Van den Bossche J. Immunometabolism in the single-cell era. Cell Metab. 2020;32:710-25.

233. Evers TMJ, Hochane M, Tans SJ, Heeren R, Semrau S, Nemes $P$, et al. Deciphering metabolic heterogeneity by single-cell analysis. Anal Chem. 2019;91:13314-23.

\section{ACKNOWLEDGEMENTS}

The following funding supports are acknowledged: NIH (R35 CA232109, PO1 DK094779, U19 Al067798, R01 Al141333, and R01 Al029564).

\section{COMPETING INTERESTS}

The authors declare no competing interests.

\section{ADDITIONAL INFORMATION}

Correspondence and requests for materials should be addressed to Wei-Chun Chou, Xin Li or Jenny P.-Y. Ting.

Reprints and permission information is available at http://www.nature.com/ reprints

OpenAccess This article is licensed under a Creative Common cc) Attribution 4.0 International License, which permits use, sharing, adaptation, distribution and reproduction in any medium or format, as long as you give appropriate credit to the original author(s) and the source, provide a link to the Creative Commons license, and indicate if changes were made. The images or other third party material in this article are included in the article's Creative Commons license, unless indicated otherwise in a credit line to the material. If material is not included in the article's Creative Commons license and your intended use is not permitted by statutory regulation or exceeds the permitted use, you will need to obtain permission directly from the copyright holder. To view a copy of this license, visit http://creativecommons. org/licenses/by/4.0/.

(c) The Author(s) 2021 